Supporting Information Available

\title{
An Efficient Route to 4-Aryl-5-pyrimidinylimidazoles via Sequential Functionalization of 2,4-Dichloropyrimidine
}

\author{
Xiaohu Deng and Neelakandha S. Mani* \\ Johnson \& Johnson Pharmaceutical Research \& Development LLC \\ 3210 Merryfield Row, San Diego, CA 92121
}

General: All the reagents were purchased from Aldrich and used without further purification. Unless otherwise specified, solvents were reagent grade and used as received. Glassware was dried in oven or flame-dried. ${ }^{1} \mathrm{H}$ and ${ }^{13} \mathrm{C}$ NMR Spectra were recorded at Bruker $500\left({ }^{1} \mathrm{H}, 500 \mathrm{MHz} ;{ }^{13} \mathrm{C}, 125 \mathrm{MHz}\right)$ or INOVA $400\left({ }^{1} \mathrm{H}, 400 \mathrm{MHz}\right.$; ${ }^{13} \mathrm{C}, 100 \mathrm{MHz}$ ) NMR Spectrometer. Infrared (IR) Spectroscopy was performed on a Nicolet Avatar 360 FT-IR. Elemental analyses were performed by NuMega Resonance Labs, Inc., San Diego, CA. HRMS (ESI) was performed on Bruker $\mu$ Tof. Flash column chromatography was performed using Merck silica gel 60 .

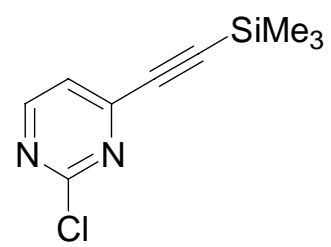

2-Chloro-4-[trimethylsilylethynyl]pyrimidine: To the solution of $\mathrm{Pd}\left(\mathrm{PPh}_{3}\right)_{2} \mathrm{Cl}_{2}(0.9 \mathrm{~g}$, 0.005 equiv) and $\mathrm{PPh}_{3}\left(0.7 \mathrm{~g}, 0.01\right.$ equiv) in a mixed solvent of THF (200 mL) and $\mathrm{Et}_{3} \mathrm{~N}$ (300 mL), 2,4-dichloropyrimidine ( $40 \mathrm{~g}, 0.27 \mathrm{~mol}, 1$ equiv) were added under $\mathrm{N}_{2}$. After bubbling $\mathrm{N}_{2}$ into the solution for $15 \mathrm{~min}, \mathrm{CuI}(0.5 \mathrm{~g}, 0.01 \mathrm{equiv})$ and trimethylsilylacetylene ( $29 \mathrm{~g}, 0.29 \mathrm{~mol}, 1.1$ equiv) were added sequentially. The reaction mixture was heated at reflux temperature for $4.5 \mathrm{~h}$ and cooled to rt. The white precipitate $\left(\mathrm{Et}_{3} \mathrm{~N} \cdot \mathrm{HCl}\right)$ was filtered off and washed with EtoAc. The filtrate solution was concentrated and the residue was diluted with hexanes and loaded directly onto a short pad of silica gel. The product was eluted with $10 \%$ EtOAc/hexanes to provide a light orange solid (49.3 g, $0.23 \mathrm{~mol}, 87 \%$ ). TLC (silica gel, Hexanes: EtOAc=4:1, $\mathrm{R}_{f}=0.42$ ). mp 51-54 ${ }^{\circ} \mathrm{C}$. IR (dry film, $\mathrm{cm}^{-1}$ ): 3125 (w), 2961 (w), 1557 (s), 1523 (s). ${ }^{1} \mathrm{H}$ NMR $\left(\mathrm{CDCl}_{3}, 400 \mathrm{MHz}, \delta\right): 8.58(\mathrm{~d}, J=5.0 \mathrm{~Hz}, 1 \mathrm{H}), 7.30(\mathrm{~d}, J=5.0 \mathrm{~Hz}, 1 \mathrm{H}), 0.28(\mathrm{~s}, 9 \mathrm{H})$. ${ }^{13} \mathrm{C} \mathrm{NMR}\left(\mathrm{CDCl}_{3}, 100 \mathrm{MHz}, \delta\right): 161.78,159.71,152.97,122.08,103.79,100.31 .-0.44$. Anal. Calcd for $\mathrm{C}_{9} \mathrm{H}_{11} \mathrm{ClN}_{2} \mathrm{Si}$ : C, 51.29; H, 5.26; N, 13.29; Found: C, 51.45; H, 5.16; N, 13.33. 


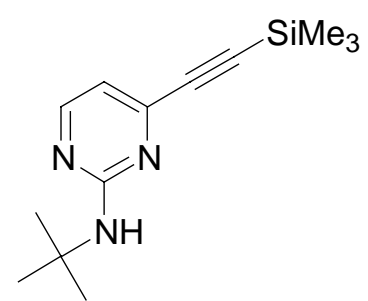

2-tert-Butylamino-4-[trimethylsilylethynyl]pyrimidine: In a sealed tube, 2-chloro-4[trimethylsilylethynyl]pyrimidine $(25 \mathrm{~g}, 0.12 \mathrm{~mol})$ in tert-butylamine $(100 \mathrm{~mL})$ was heated at $80{ }^{\circ} \mathrm{C}$ for 2 days. The mixture was cooled to rt and triturated with hexanes (100 $\mathrm{mL})$. The resulting white precipitate was filtered and washed with hexanes. The filtrate was concentrated to the give the crude product as a brown oil, which was used in the following reaction without further purification. TLC (silica gel, Hexanes:EtOAc $=4: 1, \mathrm{R}_{f}$ $=0.40$ ); mp 71-73 ${ }^{\circ} \mathrm{C}$; IR (dry film, cm ${ }^{-1}$ ): 3293 (br, w), 2962 (w), 1569 (s). ${ }^{1} \mathrm{H} \mathrm{NMR}$ $\left(\mathrm{CDCl}_{3}, 400 \mathrm{MHz}, \delta\right): 8.22(\mathrm{~d}, J=5.0 \mathrm{~Hz}, 1 \mathrm{H}), 6.57(\mathrm{~d}, J=5.0 \mathrm{~Hz}, 1 \mathrm{H}), 5.19(\mathrm{~s}, 1 \mathrm{H})$, $1.42(\mathrm{~s}, 9 \mathrm{H}), 0.26(\mathrm{~s}, 9 \mathrm{H}) .{ }^{13} \mathrm{C} \mathrm{NMR}\left(\mathrm{CDCl}_{3}, 100 \mathrm{MHz}, \delta\right): 162.20,157.96,150.76$, 112.75, 102.58, 97.43, 51.22, 29.06, -0.19. Anal. Calcd for $\mathrm{C}_{13} \mathrm{H}_{21} \mathrm{~N}_{3} \mathrm{Si}: \mathrm{C}, 63.11 ; \mathrm{H}$, 8.56; N, 16.98; Found: C, 63.20; H, 8.52; N, 16.70.

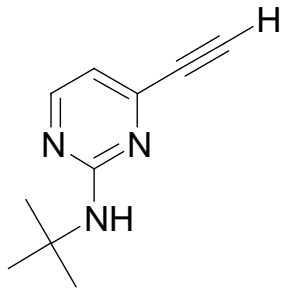

tert-Butylamino-4-ethynylpyrimidine: A solution of 2-tert-butylamino-4[trimethylsilylethynyl]pyrimidine in $\mathrm{CH}_{3} \mathrm{OH}(150 \mathrm{~mL})$ was treated with a solution of $\mathrm{KOH}(30 \mathrm{mg}, 0.005$ equiv) in $5 \mathrm{~mL} \mathrm{CH} 3 \mathrm{OH}$. After $30 \mathrm{~min}$, additional $\mathrm{KOH}(30 \mathrm{mg}$, 0.005 equiv) in $5 \mathrm{~mL} \mathrm{CH} \mathrm{CH}_{3} \mathrm{OH}$ was added. After a further $30 \mathrm{~min}$, the solvent was evaporated and the residue was re-dissolved in hexanes and loaded directly onto a short pad of silica gel. The product was eluted with $10 \%$ EtOAc in hexanes to provide the crude product as a light brown oil (18.6 g, $0.11 \mathrm{~mol}, 90 \%, 2$ steps). TLC (silica gel, Hexanes:EtOAc = 4:1, $\left.\mathrm{R}_{f}=0.29\right)$. IR (dry film, $\left.\mathrm{cm}^{-1}\right): 3294(\mathrm{~m}), 2965(\mathrm{~m}), 2114(\mathrm{w})$, 1571 (s). ${ }^{1} \mathrm{H} \mathrm{NMR}\left(\mathrm{CDCl}_{3}, 400 \mathrm{MHz}, \delta\right): 8.23(\mathrm{~d}, J=5.0 \mathrm{~Hz}, 1 \mathrm{H}), 6.60(\mathrm{~d}, J=5.0 \mathrm{~Hz}$, $1 \mathrm{H}), 5.20(\mathrm{~s}, 1 \mathrm{H}), 3.15(\mathrm{~s}, 1 \mathrm{H}), 1.43(\mathrm{~s}, 9 \mathrm{H}) .{ }^{13} \mathrm{C} \mathrm{NMR}\left(\mathrm{CDCl}_{3}, 100 \mathrm{MHz}, \delta\right): 162.17$, 158.18, 150.07, 112.82, 81.90, 78.91, 51.25, 29.00. HRMS (ESI) Calcd for $\mathrm{C}_{10} \mathrm{H}_{14} \mathrm{~N}_{3}$ $[\mathrm{M}+\mathrm{H}]^{+}:$176.1182. Found: 176.1189.

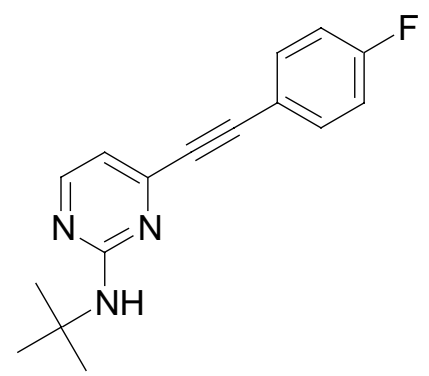

2-tert-Butylamino-4-[4-fluoro-phenylethynyl]pyrimidine:, To the solution of $\mathrm{Pd}\left(\mathrm{PPh}_{3}\right) \mathrm{Cl}_{2}\left(0.4 \mathrm{~g}, 0.005\right.$ equiv) in $\mathrm{Et}_{3} \mathrm{~N}(150 \mathrm{~mL})$ and THF $(150 \mathrm{~mL})$, was added tert- 
butylamino-4-ethynylpyrimidine ( $18.6 \mathrm{~g}, 0.11 \mathrm{~mol}, 1$ equiv) under $\mathrm{N}_{2}$. The solution was bubbled with $\mathrm{N}_{2}$ for $15 \mathrm{~min}$ and then 4-iodo-fluorobenzene (23.6 g, $0.11 \mathrm{~mol}, 1$ equiv) and $\mathrm{CuI}(0.22 \mathrm{~g}, 0.01$ equiv) were added sequentially. The reaction mixture was stirred at rt for $2.5 \mathrm{~h}$. The precipitate $\left(\mathrm{Et}_{3} \mathrm{~N} \cdot \mathrm{HCl}\right)$ was filtered and washed with EtOAc. The filtrated was concentrated and the residue was diluted with EtOAc and loaded directly on a short pad of silica gel. The product was eluted with $10 \%$ EtOAc in hexanes to provide the crude product, which was recrystallized from EtOAc/hexanes to give the title compound as a light yellow solid $(25 \mathrm{~g}, 0.093 \mathrm{~mol}, 84 \%)$. TLC (silica gel, Hexanes: EtOAc $\left.=4: 1, \mathrm{R}_{f}=0.30\right) . \mathrm{mp} 97-99^{\circ} \mathrm{C} . \mathrm{IR}\left(\operatorname{dry}\right.$ film, $\left.\mathrm{cm}^{-1}\right): 3287(\mathrm{w}), 2964(\mathrm{w}), 2222(\mathrm{w})$, $1566(\mathrm{~s}) .{ }^{1} \mathrm{H} \mathrm{NMR}\left(\mathrm{CDCl}_{3}, 500 \mathrm{MHz}, \delta\right): 8.26(\mathrm{~d}, J=4.2 \mathrm{~Hz}, 1 \mathrm{H}), 7.60-7.55(\mathrm{~m}, 2 \mathrm{H})$, 7.10-7.00 (m, 2H), $6.65(\mathrm{~d}, J=4.9 \mathrm{~Hz}, 1 \mathrm{H}), 5.22(\mathrm{~s}, 1 \mathrm{H}), 1.45(\mathrm{~s}, 9 \mathrm{H}) .{ }^{13} \mathrm{C} \mathrm{NMR}\left(\mathrm{CDCl}_{3}\right.$, $125 \mathrm{MHz}, \delta): 163.20\left(\mathrm{~d}, J_{C-F}=250 \mathrm{~Hz}\right), 162.24,157.98,151.04,134.53\left(\mathrm{~d}, J_{C-F}=8.5\right.$ $\mathrm{Hz}), 118.04\left(\mathrm{~d}, J_{C-F}=3.5 \mathrm{~Hz}\right), 116.06\left(\mathrm{~d}, J_{C-F}=22 \mathrm{~Hz}\right), 112.58,90.12,87.45,51.27$, 29.07. Anal. Calcd for $\mathrm{C}_{16} \mathrm{H}_{16} \mathrm{FN}_{3}$ : C, 71.36; H, 5.99; N, 15.60; Found: $\mathrm{C}, 71.25$; $\mathrm{H}$, $5.95 ; \mathrm{N}, 15.66$.

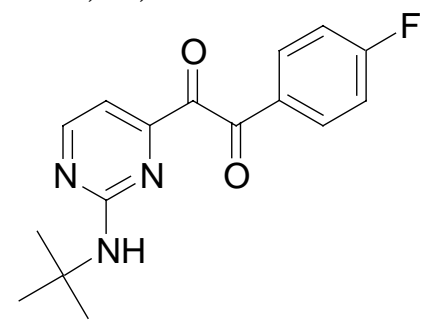

1-(2-tert-Butylamino-pyrimidin-4-yl)-2-(4-fluoro-phenyl)-ethane-1,2-dione: To the solution of 2-tert-butylamino-4-[4-fluoro-phenylethynyl]pyrimidine (5.0 g, $19 \mathrm{mmol}, 1$ equiv) in $225 \mathrm{~mL}$ acetone, was added the solution of $\mathrm{NaHCO}_{3}(0.40 \mathrm{~g})$ and $\mathrm{MgSO}_{4}(4.0$ g) in $120 \mathrm{~mL} \mathrm{H}_{2} \mathrm{O}$. Under vigorous stirring, $\mathrm{KMnO}_{4}$ (Cairox $\mathrm{M}$ from Carux Co., 325 mesh, $11.1 \mathrm{~g}, 70 \mathrm{mmol}, 3.8$ equiv) was added at $\mathrm{rt}$ in one portion. After $4 \mathrm{~min}$, the reaction was quenched by the addition of $\mathrm{NaHSO}_{3}(15 \mathrm{~g})$ in $30 \mathrm{~mL} \mathrm{H}_{2} \mathrm{O}$. After stirring for $10 \mathrm{~min}$, the solution was acidified with $50 \% \mathrm{H}_{2} \mathrm{SO}_{4}$ to $\mathrm{pH}<2$ and extracted with 1:1 $\mathrm{Et}_{2} \mathrm{O} /$ hexanex $(300 \mathrm{~mL})$ and then EtOAc $(50 \mathrm{~mL})$. The combined organic layer was washed with aq. $\mathrm{K}_{2} \mathrm{CO}_{3}(1 \mathrm{~mol} / \mathrm{L}, 100 \mathrm{~mL}$, saturated with $\mathrm{NaCl})$ and brine $(100 \mathrm{~mL})$, dried $\left(\mathrm{Na}_{2} \mathrm{SO}_{4}\right)$ and concentrated to provide the title compound as a light yellow solid (4.0 g, $13 \mathrm{mmol}, 71 \%)$. TLC (silica gel, Hexanes: EtOAc=4:1, $\mathrm{R}_{f}=0.15$ ). $\mathrm{mp} 123-124$ ${ }^{\circ} \mathrm{C}$. IR (dry film, cm ${ }^{-1}$ ): 3297 (br w), 2968 (w), 1711 (m) 1680 (m), 1596 (s), 1583 (s). ${ }^{1} \mathrm{H}$ NMR $\left(\mathrm{CDCl}_{3}, 400 \mathrm{MHz}, \delta\right): 8.54(\mathrm{~d}, J=3.4 \mathrm{~Hz}, 1 \mathrm{H}), 8.0-7.90(\mathrm{~m}, 2 \mathrm{H}), 7.22-7.12(\mathrm{~m}$, $3 \mathrm{H}), 5.29$ (s, 1H), $1.18(\mathrm{~s}, 9 \mathrm{H}) .{ }^{13} \mathrm{C} \mathrm{NMR}\left(\mathrm{CDCl}_{3}, 100 \mathrm{MHz}, \delta\right): 195.42,194.87,166.82$ $\left(\mathrm{d}, J_{C-F}=256 \mathrm{~Hz}\right), 162.01,160.58,157.76,132.28\left(\mathrm{~d}, J_{C-F}=9.6 \mathrm{~Hz}\right), 129.92\left(\mathrm{~d}, J_{C-F}=2.8\right.$ $\mathrm{Hz}), 116.55\left(\mathrm{~d}, J_{C-F}=22 \mathrm{~Hz}\right), 106.63,51.31,28.46$. Anal. Calcd for $\mathrm{C}_{16} \mathrm{H}_{16} \mathrm{FN}_{3} \mathrm{O}_{2}: \mathrm{C}$, 63.78; H, 5.35; N, 13.95; Found: C, 63.85; H, 5.22; N, 13.94.

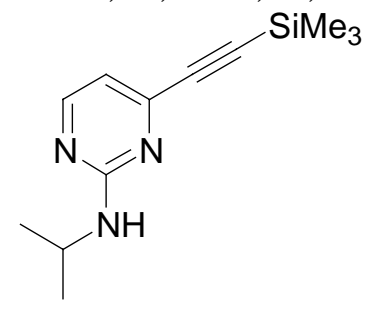


2-iso-Propylamino-4-[trimethylsilylethynyl]pyrimidine: Following the procedure described on 2-tert-butylamino-4-[trimethylsilylethynyl]pyrimidine, the title compound was obtained as a brown solid and was used on the following reaction without further purification. TLC (silica gel, Hexanes: EtOAc $=4: 1, \mathrm{R}_{f}=0.31$ ). mp 93-94 ${ }^{\circ} \mathrm{C}$. IR (dry film, $\mathrm{cm}^{-1}$ ): 3273 (br w), 2967 (m), 1570 (s). ${ }^{1} \mathrm{H} \mathrm{NMR}\left(\mathrm{CDCl}_{3}, 400 \mathrm{MHz}, \delta\right): 8.23$ (d, $J=$ $5.0 \mathrm{~Hz}, 1 \mathrm{H}), 6.59(\mathrm{~d}, J=5.0 \mathrm{~Hz}, 1 \mathrm{H}), 5.02(\mathrm{~d}, J=5.5 \mathrm{~Hz}, 1 \mathrm{H}), 4.14$ (sept, $J=6.4 \mathrm{~Hz}$, $1 \mathrm{H}), 1.21(\mathrm{~d}, J=6.4 \mathrm{~Hz}, 6 \mathrm{H}), 0.25(\mathrm{~s}, 9 \mathrm{H}) .{ }^{13} \mathrm{C} \mathrm{NMR}\left(\mathrm{CDCl}_{3}, 100 \mathrm{MHz}, \delta\right): 161.86$, 158.47, 151.11, 113.00, 102.48, 97.93, 42.98, 23.06, -0.21. HRMS (ESI) Calcd for $\mathrm{C}_{12} \mathrm{H}_{20} \mathrm{~N}_{3} \mathrm{Si}[\mathrm{M}+\mathrm{H}]^{+}: 234.1421$. Found: 234.1409.

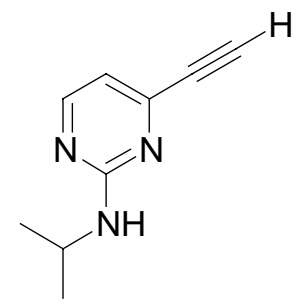

2-iso-Propylamino-4-ethynylpyrimidine: Following the procedure described on tertbutylamino-4-ethynylpyrimidine, the title compound was obtained as a light brown solid in $92 \%$ yield over 2 steps. TLC (silica gel, Hexanes:EtOAc $=4: 1, \mathrm{R}_{f}=0.24$ ). $\mathrm{mp} 84-85$ ${ }^{\circ} \mathrm{C}$. IR (dry film, $\mathrm{cm}^{-1}$ ): 3256 (br, m), 2974 (w), 2113 (w), 1568 (s). ${ }^{1} \mathrm{H} \mathrm{NMR}\left(\mathrm{CDCl}_{3}\right.$, $400 \mathrm{MHz}, \delta): 8.25$ (d, $J=5.0 \mathrm{~Hz}, 1 \mathrm{H}), 6.62(\mathrm{~d}, J=5.0 \mathrm{~Hz}, 1 \mathrm{H}), 5.05$ (s, 1H), 4.14 (sept, $J$ $=6.5 \mathrm{~Hz}, 1 \mathrm{H}), 3.19(\mathrm{~s}, 1 \mathrm{H}), 1.22(\mathrm{~d}, J=6.5 \mathrm{~Hz}, 6 \mathrm{H}) .{ }^{13} \mathrm{C} \mathrm{NMR}\left(\mathrm{CDCl}_{3}, 100 \mathrm{MHz}, \delta\right)$ : 161.88, 158.67, 150.49, 113.04, 81.80, 79.37, 43.01, 23.02. HRMS (ESI) Calcd for $\mathrm{C}_{9} \mathrm{H}_{12} \mathrm{~N}_{3}[\mathrm{M}+\mathrm{H}]^{+}:$162.1026. Found: 162.1017 .

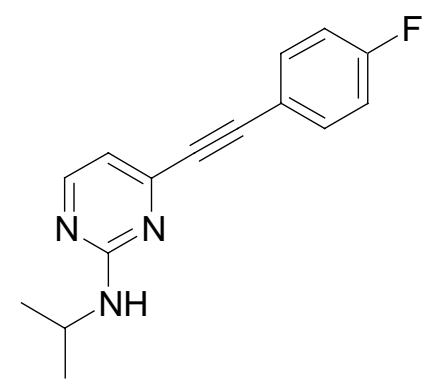

2-iso-Propylamino-4-[4-fluoro-phenylethynyl]pyrimidine: Following the procedure described on 2-tert-butylamino-4-[4-fluoro-phenylethynyl]pyrimidine, the title compound was obtained as a light yellow solid in $89 \%$ yield. TLC (silica gel, Hexanes:EtOAc =

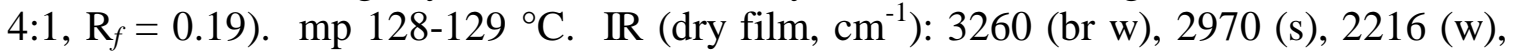
$1564(\mathrm{~s}) .{ }^{1} \mathrm{H} \mathrm{NMR}\left(\mathrm{CDCl}_{3}, 500 \mathrm{MHz}, \delta\right): 8.28(\mathrm{~d}, J=4.6 \mathrm{~Hz}, 1 \mathrm{H}), 7.60-7.50(\mathrm{~m}, 2 \mathrm{H})$, 7.10-7.00 (m, 2H), $6.66(\mathrm{~d}, J=4.9 \mathrm{~Hz}, 1 \mathrm{H}), 5.05(\mathrm{~d}, J=7.4 \mathrm{~Hz}, 1 \mathrm{H}), 4.17$ (sept, $J=6.5$ $\mathrm{Hz}, 1 \mathrm{H}), 1.24(\mathrm{~d}, J=6.5 \mathrm{~Hz}, 6 \mathrm{H}) .{ }^{13} \mathrm{C} \mathrm{NMR}\left(\mathrm{CDCl}_{3}, 125 \mathrm{MHz}\right): \delta 163.44\left(\mathrm{~d}, J_{C-F}=250\right.$ $\mathrm{Hz}), 161.92,158.47,151.44,134.55\left(\mathrm{~d}, J_{C-F}=8.5 \mathrm{~Hz}\right), 117.96\left(\mathrm{~d}, J_{C-F}=3.4 \mathrm{~Hz}\right), 116.07$ $\left(\mathrm{d}, J_{C-F}=22 \mathrm{~Hz}\right), 112.82,90.57,87.39,43.05,23.09$. Anal. Calcd for $\mathrm{C}_{15} \mathrm{H}_{14} \mathrm{FN}_{3}$ : C, 70.57; H, 5.53; N, 16.46; Found: C, 70.60; H, 5.42; N, 16.45 . 
<smiles>CC(C)Nc1nccc(C(=O)C(=O)c2ccc(F)cc2)n1</smiles>

1-(2-iso-Propylamino-pyrimidin-4-yl)-2-(4-fluoro-phenyl)-ethane-1,2-dione:

Following the procedure described on 1-(2-tert-Butylamino-pyrimidin-4-yl)-2-(4-fluorophenyl)-ethane-1,2-dione, the title compound was obtained as a light greenish solid in $56 \%$ yield. TLC (silica gel, Hexanes:EtOAc $=4: 1, \mathrm{R}_{f}=0.14$ ). $\mathrm{mp} 75-77{ }^{\circ} \mathrm{C}$. IR (dry film, $\mathrm{cm}^{-1}$ ): 3275 (br. w), 2971 (w), 1710 (m), 1682 (m). ${ }^{1} \mathrm{H} \mathrm{NMR}\left(\mathrm{CDCl}_{3}, 500 \mathrm{MHz}, \delta\right)$ : 8.57 (s, 1H), 8.00-7.90 (m, 2H), 7.23-7.15 (m, 3H), 5.08 (s, 1H), 3.73 (br. s, 1H), 1.09 (s, $6 \mathrm{H}) .{ }^{13} \mathrm{C} \mathrm{NMR}\left(\mathrm{CDCl}_{3}, 125 \mathrm{MHz}, \delta\right): 166.83\left(\mathrm{~d}, J_{C-F}=256 \mathrm{~Hz}\right), 161.87,160.67,158.29$, $132.34\left(\mathrm{~d}, J_{C-F}=8.8 \mathrm{~Hz}\right), 129.99\left(\mathrm{~d}, J_{C-F}=3.9 \mathrm{~Hz}\right), 116.53\left(\mathrm{~d}, J_{C-F}=22 \mathrm{~Hz}\right), 106.91$, 43.4122 .50 . (certain carbon signals were too weak to be detected) Anal. Calcd for $\mathrm{C}_{15} \mathrm{H}_{14} \mathrm{FN}_{3} \mathrm{O}_{2}$ : C, 62.71; H, 4.91; N, 14.63; Found: C, 62.90; H, 4.84; N, 14.76.

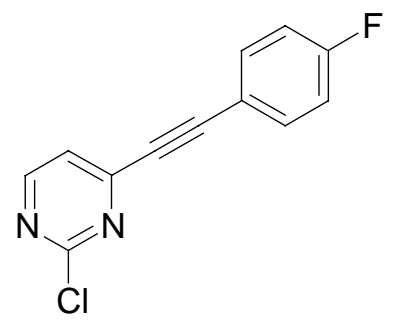

2-Chloro-4-(4-fluoro-phenylethynyl)-pyrimidine: Following the procedure described on 2-tert-butylamino-4-[trimethylsilylethynyl]pyrimidine, the title compound was prepared from 2,4-dichloropyrmidine and 1-ethynyl-4-fluorobenzene in $65 \%$ yield. TLC (silica gel, Hexanes:EtOAc $\left.=4: 1, \mathrm{R}_{f}=0.21\right) . \mathrm{mp} 125-126^{\circ} \mathrm{C} . \mathrm{IR}\left(\operatorname{dry}\right.$ film, $\left.\mathrm{cm}^{-1}\right)$ : 3049 (w), $2210(\mathrm{w}), 1559$ (s). ${ }^{1} \mathrm{H} \mathrm{NMR}\left(\mathrm{CDCl}_{3}, 500 \mathrm{MHz}, \delta\right): 8.61(\mathrm{~d}, J=5.0 \mathrm{~Hz}, 1 \mathrm{H}), 7.70-$

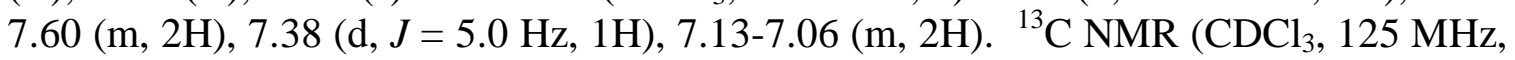
$\delta): 163.99\left(\mathrm{~d}, J_{C-F}=252 \mathrm{~Hz}\right), 161.86,159.66,153.39,134.96\left(\mathrm{~d}, J_{C-F}=8.6 \mathrm{~Hz}\right), 121.77$, $116.96\left(\mathrm{~d}, J_{C-F}=3.4 \mathrm{~Hz}\right), 116.40\left(\mathrm{~d}, J_{C-F}=22 \mathrm{~Hz}\right), 95.27,85.89$. Anal. Calcd for $\mathrm{C}_{12} \mathrm{H}_{6} \mathrm{ClFN}_{2}$ : C, 61.95; H, 2.60; N, 12.04; Found: C, 62.15; H, 2.83; N, 12.00.<smiles>CC(C)(C)N/C(=C\c1ccnc(Cl)n1)c1ccc(F)cc1</smiles>

tert-Butyl-[2-(2-chloro-pyrimidin-4-yl)-1-(4-fluoro-phenyl)-vinyl]-amine: In a sealed tube, 2-chloro-4-(4-fluoro-phenylethynyl)-pyrimidine (400 mg, $1.7 \mathrm{mmol}, 1$ equiv) in tert-butylamine $(5 \mathrm{~mL})$ was heated at $80{ }^{\circ} \mathrm{C}$ for $16 \mathrm{~h}$. After removal of tert-butylamine by evaporation, the crude product was purified on silica gel to afford tert-butylamino-4[4-fluoro-phenylethynyl]pyrimidine $(0.16 \mathrm{~g}, 34 \%)$ and the title compound $(0.29 \mathrm{~g}, 55 \%)$. TLC (silica gel, Hexanes:EtOAc $\left.=4: 1, \mathrm{R}_{f}=0.36\right) . \mathrm{mp} 152-153{ }^{\circ} \mathrm{C}$. IR $\left(\right.$ dry film, $\left.\mathrm{cm}^{-1}\right)$ : 2970 (w), 1615 (w), 1569 (s). ${ }^{1} \mathrm{H}$ NMR $\left(\mathrm{CDCl}_{3}, 500 \mathrm{MHz}, \delta\right): 10.01$ (s, 1H), 8.10 (d, J = 
$5.5 \mathrm{~Hz}, 1 \mathrm{H}), 7.40-7.34(\mathrm{~m}, 2 \mathrm{H}), 7.10-7.05(\mathrm{~m}, 2 \mathrm{H}), 6.57(\mathrm{~d}, J=5.5 \mathrm{~Hz}, 1 \mathrm{H}), 4.82(\mathrm{~s}, 1 \mathrm{H})$, $1.20(\mathrm{~s}, 9 \mathrm{H}) .{ }^{13} \mathrm{C} \mathrm{NMR}\left(\mathrm{CDCl}_{3}, 125 \mathrm{MHz}, \delta\right): 166.54,163.19\left(\mathrm{~d}, J_{C-F}=247 \mathrm{~Hz}\right), 161.25$, 159.44, 155.96, $135.08\left(\mathrm{~d}, J_{C-F}=3.6 \mathrm{~Hz}\right), 130.69\left(\mathrm{~d}, J_{C-F}=8.3 \mathrm{~Hz}\right), 115.60,115.04(\mathrm{~d}$, $J_{C-F}=22 \mathrm{~Hz}$ ), 96.01, 54.57, 32.14. Anal. Calcd for $\mathrm{C}_{16} \mathrm{H}_{17} \mathrm{ClFN}_{3}: \mathrm{C}, 62.85 ; \mathrm{H}, 5.60 ; \mathrm{N}$, 13.74; Found: $\mathrm{C}, 62.92 ; \mathrm{H}, 5.62 ; \mathrm{N}, 13.88$. The geometry of the double bond was assigned based on NOE study.

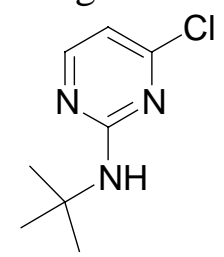

tert-Butyl-(4-chloro-pyrimidin-2-yl)-amine: A solution of 2,4-dicholoropyrimidine (10 $\mathrm{g}, 67 \mathrm{mmol})$ in tert-butylamine $(100 \mathrm{~mL})$ was refluxed for 3 hours. After removal of tertbutylamine by evaporation, the crude product was purified on silica gel to afford the title compound (3.4 g, 26\%) and tert-butyl-(2-chloro-pyrimidin-4-yl)-amine $(8.1 \mathrm{~g}, 65 \%)$. TLC (silica gel, Hexanes:EtOAc $\left.=4: 1, \mathrm{R}_{f}=0.07\right) . \mathrm{mp} 55-56^{\circ} \mathrm{C} . \quad$ IR $\left(\right.$ dry film, $\mathrm{cm}^{-1}$ ): 3280 (br w), 2966 (w), 1577 (s). ${ }^{1} \mathrm{H}$ NMR $\left(\mathrm{CDCl}_{3}, 400 \mathrm{MHz}, \delta\right): 8.10$ (d, $J=5.0 \mathrm{~Hz}$, $1 \mathrm{H}), 6.52(\mathrm{~d}, J=5.0 \mathrm{~Hz}, 1 \mathrm{H}), 5.36(\mathrm{~s}, 1 \mathrm{H}), 1.44(\mathrm{~s}, 9 \mathrm{H}) .{ }^{13} \mathrm{C} \mathrm{NMR}\left(\mathrm{CDCl}_{3}, 100 \mathrm{MHz}, \delta\right)$ : 161.81, 161.08, 158.50, 109.69, 51.63, 28.90. Anal. Calcd for $\mathrm{C}_{8} \mathrm{H}_{12} \mathrm{ClN}_{3}$ : C, 51.76; $\mathrm{H}$, $6.51 ; \mathrm{N}, 22.63$; Found: C, 51.78; H, 6.56; N, 22.81.<smiles>CC(C)(C)Nc1ccnc(Cl)n1</smiles>

tert-Butyl-(2-chloro-pyrimidin-4-yl)-amine: TLC (silica gel, Hexanes:EtOAc $=4: 1, \mathrm{R}_{f}$ $=0.34$ ). $\mathrm{mp} 104-105{ }^{\circ} \mathrm{C}$. IR (dry film, $\mathrm{cm}^{-1}$ ): 3268 (br. w), 2967 (w), 1588 (s). ${ }^{1} \mathrm{H}$ NMR $\left(\mathrm{CDCl}_{3}, 400 \mathrm{MHz}, \delta\right): 7.96(\mathrm{~d}, J=5.5 \mathrm{~Hz}, 1 \mathrm{H}), 6.25(\mathrm{~d}, J=5.2 \mathrm{~Hz}, 1 \mathrm{H}), 5.10(\mathrm{~s}, 1 \mathrm{H})$, 1.44 (s, 9H). ${ }^{13} \mathrm{C} \mathrm{NMR}\left(\mathrm{CDCl}_{3}, 100 \mathrm{MHz}, \delta\right): 163.10,160.60,156.51,103.97,52.09$, 29.03. Anal. Calcd for $\mathrm{C}_{8} \mathrm{H}_{12} \mathrm{ClN}_{3}$ : C, 51.76; H, 6.51; N, 22.63; Found: C, 51.74; H, $6.55 ; \mathrm{N}, 22.80$.

\section{Procedure for Imidazole Synthesis:}<smiles>CC(C)Nc1nccc(-c2ncc[nH]2)c1-c1ccc(F)cc1</smiles>

iso-Propyl-\{4-[5-(4-fluoro-phenyl)-1H-imidazol-4-yl]-pyrimidin-2-yl\}-amine: A mixture of 1-(2-iso-propylamino-pyrimidin-4-yl)-2-(4-fluoro-phenyl)-ethane-1,2-dione (12.5 g, $43.5 \mathrm{mmol}, 1.0$ equiv.), hexamethylenetetramine (12.2 g, $87.1 \mathrm{mmol}, 2.0$ equiv.), $\mathrm{NH}_{4} \mathrm{OAc}\left(17 \mathrm{~g}, 220 \mathrm{mmol}, 5\right.$ equiv.), and $\mathrm{Na}_{2} \mathrm{SO}_{4}(12 \mathrm{~g})$ in $\mathrm{AcOH}(150 \mathrm{~mL})$ was heated 
at $65{ }^{\circ} \mathrm{C}$ for 4 hours and then cooled to room temperature. The solid was filtered off and washed with $\mathrm{AcOH}$. The filtrate solution was concentrated and the residue was redissolved in $\mathrm{CH}_{2} \mathrm{Cl}_{2}$. After washed with aq. $\mathrm{NaOH}$ solution $(1 \mathrm{~mol} / \mathrm{L})$, the organic layer was dried $\left(\mathrm{Na}_{2} \mathrm{SO}_{4}\right)$, concentrated and then purified by column chromatography (MeOH/hexanes) to afford the title compound as a light yellow solid $(6.8 \mathrm{~g}, 22.9 \mathrm{mmol}$, $52 \%$ ). TLC (silica gel, $10 \% \mathrm{MeOH}$ in $\mathrm{CH}_{2} \mathrm{Cl}_{2}, \mathrm{R}_{f}=0.21$ ). IR (dry film, $\mathrm{cm}^{-1}$ ): $2968(\mathrm{w}$ ), 1568 (s), 1502 (s), 1224 (m). ${ }^{1} \mathrm{H} \mathrm{NMR}\left(\mathrm{CDCl}_{3}, 500 \mathrm{MHz}, \delta\right): 8.13$ (br. s, $\left.1 \mathrm{H}\right), 7.84$ (br. s, $1 \mathrm{H}$ ), 7.61 (br. s, 2H), 7.14 (t, $J=7.8 \mathrm{~Hz}, 2 \mathrm{H}$ ), 6.87 (br. s, 1H), 5.31 (br. s, $1 \mathrm{H}$ ), 4.14 (br. $\mathrm{s}, 1 \mathrm{H}), 1.26(\mathrm{~d}, J=9.8 \mathrm{~Hz}, 6 \mathrm{H}) \cdot{ }^{13} \mathrm{C} \mathrm{NMR}\left(\mathrm{CDCl}_{3}, 125 \mathrm{MHz}, \delta\right): 162.96\left(\mathrm{~d}, J_{C-F}=247\right.$ $\mathrm{Hz}), 161.45,157.83,157.49,131.20\left(\mathrm{~d}, J_{C-F}=8.0 \mathrm{~Hz}\right), 130.16,115.66\left(\mathrm{~d}, J_{C-F}=22 \mathrm{~Hz}\right)$, 105.82, 42.93, 23.00. HRMS (ESI) Calcd for $\mathrm{C}_{16} \mathrm{H}_{17} \mathrm{FN}_{5}[\mathrm{M}+\mathrm{H}]^{+}$: 298.1463. Found: 298.1461 .

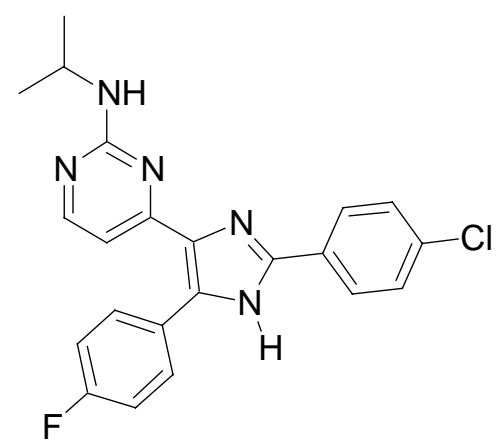

\section{\{4-[2-(4-Chloro-phenyl)-5-(4-fluoro-phenyl)-1H-imidazol-4-yl]-pyrimidin-2-yl\}-}

isopropyl-amine: Following the general procedure as shown above, the title compound was prepared from 1-(2-iso-propylamino-pyrimidin-4-yl)-2-(4-fluoro-phenyl)-ethane-1,2dione, 4-chlorobenaldehyde, and $\mathrm{NH}_{4} \mathrm{OAc}$ in $62 \%$ yield. The reaction time was 48 hours at $65{ }^{\circ} \mathrm{C}$. TLC (silica gel, Hexanes:EtOAc $\left.=1: 1, \mathrm{R}_{f}=0.44\right)$. IR (dry film, $\left.\mathrm{cm}^{-1}\right): 2968$ (w), 1569 (s), 1534 (m), 1500 (s), 1457 (m), 1219 (m). $\left.{ }^{1} \mathrm{H} \mathrm{NMR} \mathrm{(CDCl}, 500 \mathrm{MHz}, \delta\right)$ : 10.52 (br. s, $1 \mathrm{H}), 8.11(\mathrm{~d}, J=5.2 \mathrm{~Hz}, 1 \mathrm{H}), 7.87(\mathrm{~d}, J=8.5 \mathrm{~Hz}, 2 \mathrm{H}), 7.61(\mathrm{dd}, J=5.8,7.6$ $\mathrm{Hz}, 2 \mathrm{H}), 7.42$ (d, $J=8.5 \mathrm{~Hz}, 2 \mathrm{H}), 7.13(\mathrm{~d}, J=8.5 \mathrm{~Hz}, 2 \mathrm{H}), 6.61$ (br. s, $1 \mathrm{H}), 5.22$ (br. s, $1 \mathrm{H}), 4.17$ (br. s, $1 \mathrm{H}), 1.25$ (br. s, $6 \mathrm{H}) .{ }^{13} \mathrm{C} \mathrm{NMR}\left(\mathrm{CDCl}_{3}, 100 \mathrm{MHz}, \delta\right): 163.10\left(\mathrm{~d}, J_{C-F}=\right.$ $247 \mathrm{~Hz}), 161.64,158.29,145.82,135.64,131.28\left(\mathrm{~d}, J_{C-F}=8.2 \mathrm{~Hz}\right), 129.40,127.96$, 127.07, $115.77\left(\mathrm{~d}, J_{C-F}=22 \mathrm{~Hz}\right), 105.82,43.06,23.12$. HRMS (ESI) Calcd for $\mathrm{C}_{22} \mathrm{H}_{20} \mathrm{ClFN}_{5}[\mathrm{M}+\mathrm{H}]^{+}: 408.1386$. Found: 408.1404.<smiles>COC(OC)c1nc(-c2ccnc(NC(C)C)n2)c(-c2ccc(F)cc2)[nH]1</smiles> 
\{4-[2-Dimethoxymethyl-5-(4-fluoro-phenyl)-1H-imidazol-4-yl]-pyrimidin-2-yl\}-

isopropyl-amine: Following the general procedure as shown above, the title compound was prepared from 1-(2-iso-propylamino-pyrimidin-4-yl)-2-(4-fluoro-phenyl)-ethane-1,2dione, glyoxal 1,1-dimethylacetal in tert-butyl methyl ether, and $\mathrm{NH}_{4} \mathrm{OAc}$ in $58 \%$ yield. The solvent used was $\mathrm{MeOH}$ instead of $\mathrm{AcOH}$. The reaction time was 3 hours at room temperature. TLC (silica gel, Hexanes:EtOAc $\left.=1: 1, \mathrm{R}_{f}=0.12\right) . \quad \mathrm{IR}\left(\right.$ dry film, $\left.\mathrm{cm}^{-1}\right)$ : 2965 (w), 2935 (w), 1573 (s), 1515 (m), 1458 (m), 1218 (m), 1110 (s). ${ }^{1} \mathrm{H} \mathrm{NMR}\left(\mathrm{CDCl}_{3}\right.$, $500 \mathrm{MHz}, \delta): 10.31$ (br s, $1 \mathrm{H}), 8.09(\mathrm{~d}, J=5.1 \mathrm{~Hz}, 1 \mathrm{H}), 7.64-7.56(\mathrm{~m}, 2 \mathrm{H}), 7.12(\mathrm{t}, J=$ $8.6 \mathrm{~Hz}, 2 \mathrm{H}), 6.59(\mathrm{~d}, J=5.2 \mathrm{~Hz}, 1 \mathrm{H}), 5.55(\mathrm{~s}, 1 \mathrm{H}), 5.03(\mathrm{~d}, J=7.3 \mathrm{~Hz}, 1 \mathrm{H}), 4.20$ (sept, $J$ $=6.7 \mathrm{~Hz}, 1 \mathrm{H}), 3.47(\mathrm{~s}, 6 \mathrm{H}), 1.29(\mathrm{~d}, J=6.7 \mathrm{~Hz}, 6 \mathrm{H}) .{ }^{13} \mathrm{C} \mathrm{NMR}\left(\mathrm{CDCl}_{3}, 100 \mathrm{MHz}, \delta\right)$ : $163.00\left(\mathrm{~d}, J_{C-F}=246 \mathrm{~Hz}\right), 161.80,158.47,156.06,145.51,142.45,131.27\left(\mathrm{~d}, J_{C-F}=8.2\right.$ $\mathrm{Hz}), 130.91\left(\mathrm{~d}, J_{C-F}=2.1 \mathrm{~Hz}\right), 125.02,115.67\left(\mathrm{~d}, J_{C-F}=22 \mathrm{~Hz}\right), 105.77,98.41,53.91$, 43.05, 23.17. HRMS (ESI) Calcd for $\mathrm{C}_{19} \mathrm{H}_{23} \mathrm{FN}_{5} \mathrm{O}_{2}[\mathrm{M}+\mathrm{H}]^{+}:$372.1830. Found: 372.1837 .<smiles>CC(C)(C)Nc1nccc(-c2nc[nH]c2-c2ccc(F)cc2)n1</smiles>

tert-Butyl-\{4-[5-(4-fluoro-phenyl)-1H-imidazol-4-yl]-pyrimidin-2-yl\}-amine:

Following the general procedure as shown above, the title compound was prepared from 1-(2-tert-butylamino-pyrimidin-4-yl)-2-(4-fluoro-phenyl)-ethane-1,2-dione, hexamethylenetetramine, and $\mathrm{NH}_{4} \mathrm{OAc}$ in $56 \%$ yield. TLC (silica gel, $10 \% \mathrm{MeOH}$ in $\mathrm{CH}_{2} \mathrm{Cl}_{2}, \mathrm{R}_{f}=0.22$ ). IR (dry film, $\mathrm{cm}^{-1}$ ): 2957 (w), 1577 (s), 1543 9s), 1501 (s), 1220 (s). ${ }^{1} \mathrm{H} \mathrm{NMR}\left(\mathrm{CDCl}_{3}, 500 \mathrm{MHz}, \delta\right): 8.04$ (d, $\left.J=5.3 \mathrm{~Hz}, 1 \mathrm{H}\right), 7.75$ (br. s, $\left.1 \mathrm{H}\right), 7.56$ (dd, $J=$ $5.5,8.5 \mathrm{~Hz}, 2 \mathrm{H}), 7.12(\mathrm{t}, J=8.6 \mathrm{~Hz}, 2 \mathrm{H}), 6.63(\mathrm{~d}, J=5.3 \mathrm{~Hz}, 1 \mathrm{H}), 5.76$ (br. s, $1 \mathrm{H}), 1.45$ $(\mathrm{s}, 9 \mathrm{H}) .{ }^{13} \mathrm{C} \mathrm{NMR}\left(\mathrm{CDCl}_{3}, 125 \mathrm{MHz}, \delta\right): 162.99\left(\mathrm{~d}, J_{C-F}=246 \mathrm{~Hz}\right), 161.70,157.56$, 156.44, 140.65, 135.84, $131.17\left(\mathrm{~d}, J_{C-F}=8.0 \mathrm{~Hz}\right), 130.29\left(\mathrm{~d}, J_{C-F}=3.2 \mathrm{~Hz}\right), 126.36$, $115.78\left(\mathrm{~d}, J_{C-F}=21 \mathrm{~Hz}\right), 105.54,51.10,29.21$. HRMS (ESI) Calcd for $\mathrm{C}_{17} \mathrm{H}_{19} \mathrm{FN}_{5}$ $[\mathrm{M}+\mathrm{H}]^{+}:$312.1619. Found: 312.1631.

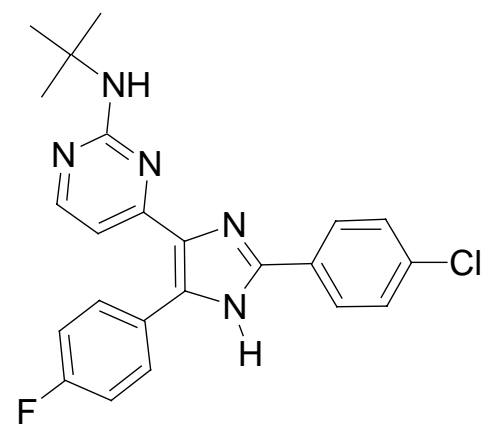


tert-Butyl-\{4-[2-(4-chloro-phenyl)-5-(4-fluoro-phenyl)-1H-imidazol-4-yl]-pyrimidin2-yl\}-amine: Following the general procedure as shown above, the title compound was prepared from 1-(2-tert-butylamino-pyrimidin-4-yl)-2-(4-fluoro-phenyl)-ethane-1,2dione, 4-chlorobenaldehyde, and $\mathrm{NH}_{4} \mathrm{OAc}$ in $68 \%$ yield. The reaction time was 48 hours at $65{ }^{\circ} \mathrm{C}$. TLC (silica gel, Hexanes:EtOAc $\left.=1: 1, \mathrm{R}_{f}=0.60\right)$. IR (dry film, $\mathrm{cm}^{-1}$ ): 2957 (w), 1565 (s), 1498 (s), 1451 (s), 1221 (s). ${ }^{1} \mathrm{H} \mathrm{NMR}\left(\mathrm{CDCl}_{3}, 500 \mathrm{MHz}, \delta\right.$ ): 10.52 (br. s, $1 \mathrm{H}), 8.10(\mathrm{~d}, J=4.7 \mathrm{~Hz}, 1 \mathrm{H}), 7.82(\mathrm{~d}, J=8.5 \mathrm{~Hz}, 2 \mathrm{H}), 7.65$ (br. s, $2 \mathrm{H}), 7.40$ (d, $J=8.3$ $\mathrm{Hz}, 2 \mathrm{H}$ ), 7.12 (br. s, 2H), 6.58 (br. s, $1 \mathrm{H}$ ), 5.29 (br. s, $1 \mathrm{H}), 1.49$ (s, 9H). ${ }^{13} \mathrm{C} \mathrm{NMR}$ $\left(\mathrm{CDCl}_{3}, 100 \mathrm{MHz}, \delta\right): 163.11\left(\mathrm{~d}, J_{C-F}=246 \mathrm{~Hz}\right), 162.08,158.20,155.31,145.69,143.49$, $135.64,131.24\left(\mathrm{~d}, J_{C-F}=8.1 \mathrm{~Hz}\right), 130.87,129.44,127.97,126.96,126.26,115.81\left(\mathrm{~d}, J_{C-F}\right.$ $=21 \mathrm{~Hz}), 105.53,51.15,29.32$. HRMS (ESI) Calcd for $\mathrm{C}_{23} \mathrm{H}_{22} \mathrm{ClFN}_{5}[\mathrm{M}+\mathrm{H}]^{+}: 422.1542$. Found: 422.1563.<smiles>COC(OC)c1nc(-c2ccnc(NC(C)(C)C)n2)c(-c2ccc(F)cc2)[nH]1</smiles>

tert-Butyl-\{4-[2-dimethoxymethyl-5-(4-fluoro-phenyl)-1H-imidazol-4-yl]-pyrimidin2-yl\}-amine: Following the general procedure as shown above, the title compound was prepared from 1-(2-tert-butylamino-pyrimidin-4-yl)-2-(4-fluoro-phenyl)-ethane-1,2dione, glyoxal 1,1-dimethylacetal in tert-butyl methyl ether, and $\mathrm{NH}_{4} \mathrm{OAc}$ in $60 \%$ yield. The solvent used was $\mathrm{MeOH}$ instead of $\mathrm{AcOH}$. The reaction time was 3 hours at room temperature. TLC (silica gel, Hexanes:EtOAc $\left.=1: 1, \mathrm{R}_{f}=0.19\right)$. IR $\left(\right.$ dry film, $\mathrm{cm}^{-1}$ ): 2953 (w), 2925 (w), 1571 (s), 1496 (m), 1452 (m), 1220 (s), 1111 (m). ${ }^{1} \mathrm{H} \mathrm{NMR}\left(\mathrm{CDCl}_{3}\right.$, $500 \mathrm{MHz}, \delta$ ): 10.29 (br. s, $1 \mathrm{H}), 8.07$ (d, $J=5.2 \mathrm{~Hz}, 1 \mathrm{H}), 7.60$ (dd, $J=5.6,8.0 \mathrm{~Hz}, 2 \mathrm{H})$, $7.11(\mathrm{t}, J=8.6 \mathrm{~Hz}, 2 \mathrm{H}), 6.57(\mathrm{~d}, J=5.2 \mathrm{~Hz}, 1 \mathrm{H}), 5.55(\mathrm{~s}, 1 \mathrm{H}), 5.23$ (br. s, $1 \mathrm{H}), 3.46$ (s, $6 \mathrm{H}), 1.49(\mathrm{~s}, 9 \mathrm{H}) .{ }^{13} \mathrm{C} \mathrm{NMR}\left(\mathrm{CDCl}_{3}, 100 \mathrm{MHz}, \delta\right): 162.97\left(\mathrm{~d}, J_{C-F}=246 \mathrm{~Hz}\right), 162.10$, $158.11,155.61,145.43,142.27,131.23\left(\mathrm{~d}, J_{C-F}=8.2 \mathrm{~Hz}\right), 130.92,125.07,115.64\left(\mathrm{~d}, J_{C-F}\right.$ $=22 \mathrm{~Hz}$ ), 105.56, 98.31, 53.83, 51.10, 29.27. HRMS (ESI) Calcd for $\mathrm{C}_{20} \mathrm{H}_{25} \mathrm{FN}_{5} \mathrm{O}_{2}$ $[\mathrm{M}+\mathrm{H}]^{+}:$386.1987. Found: 386.2005. 


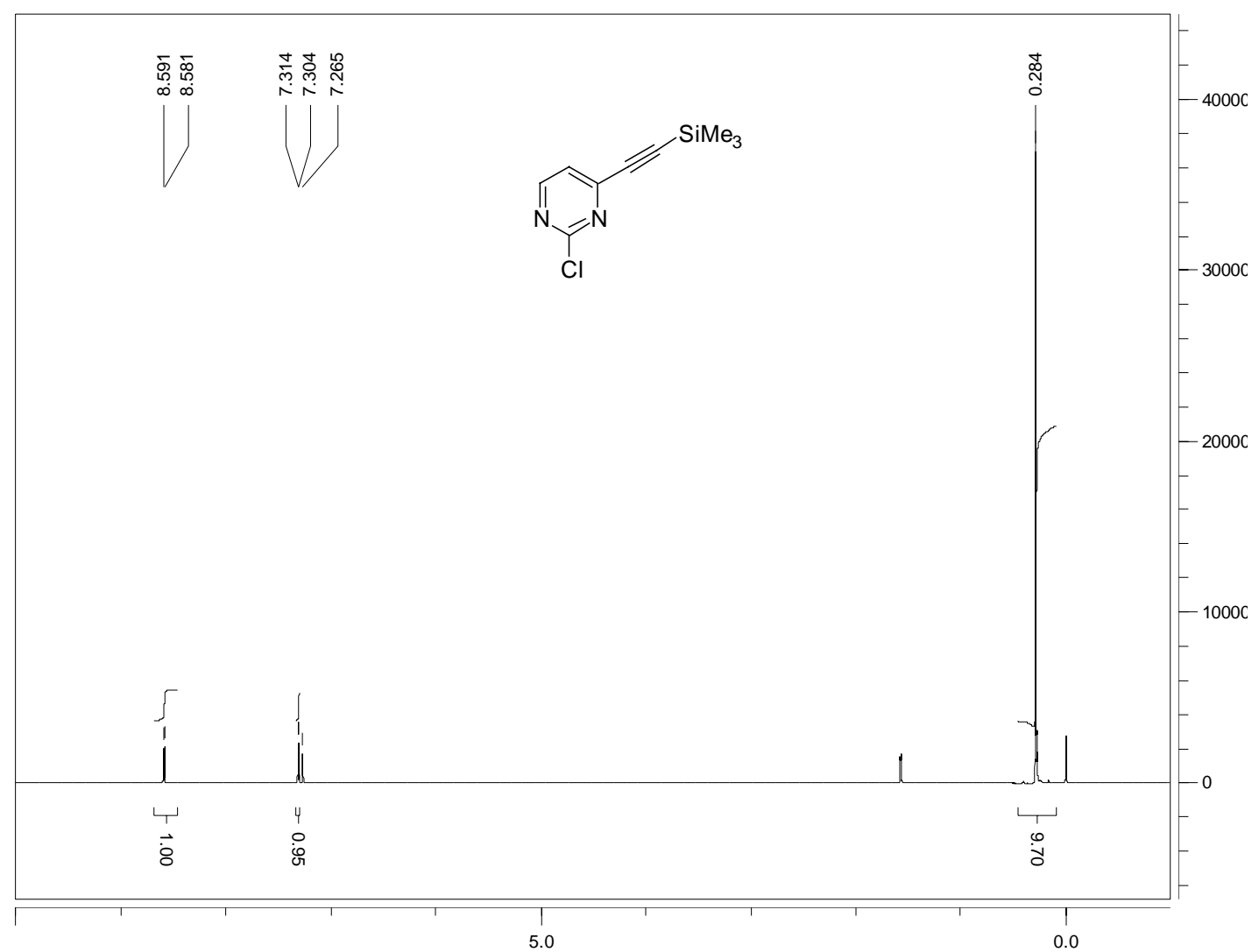

ppm (t1)

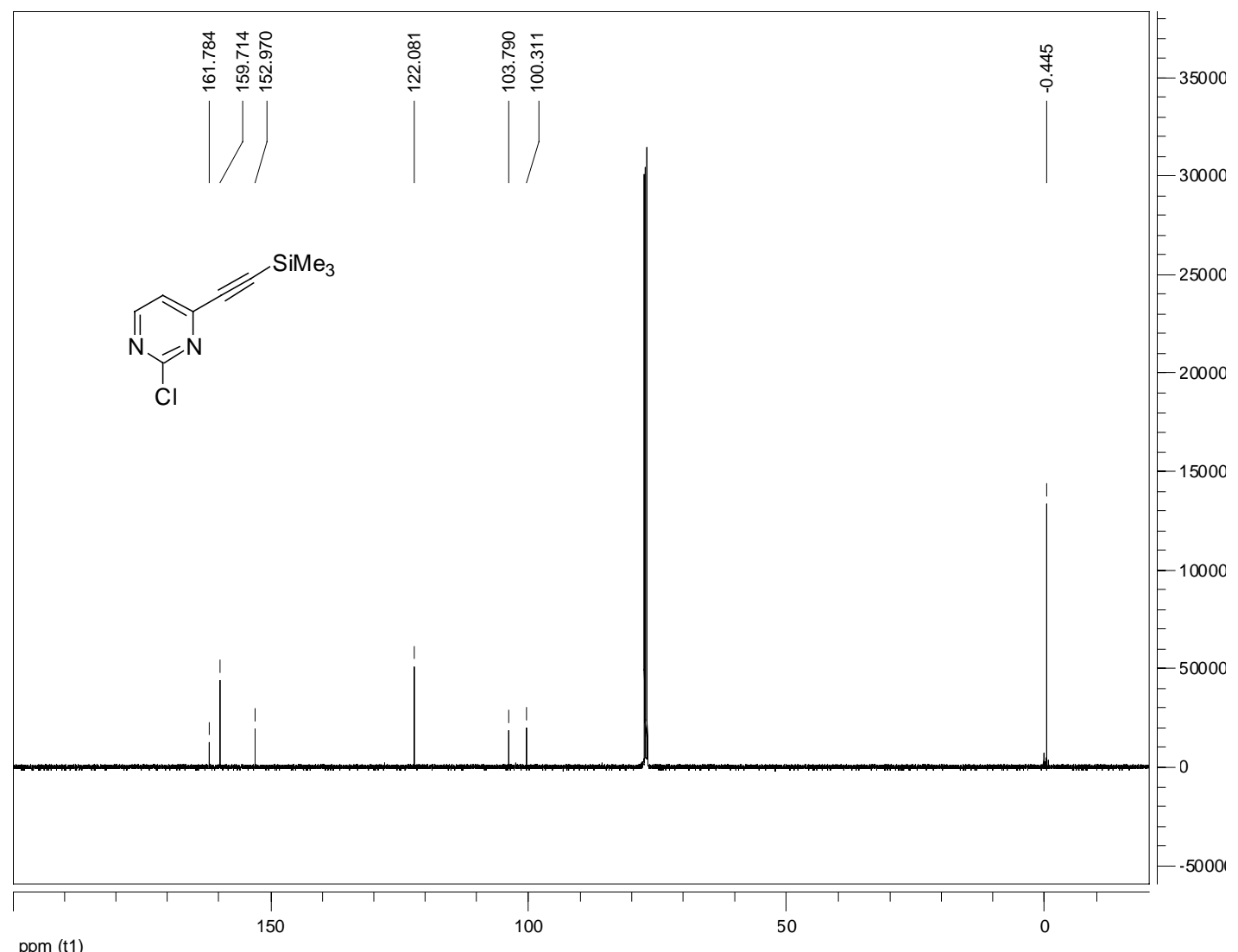




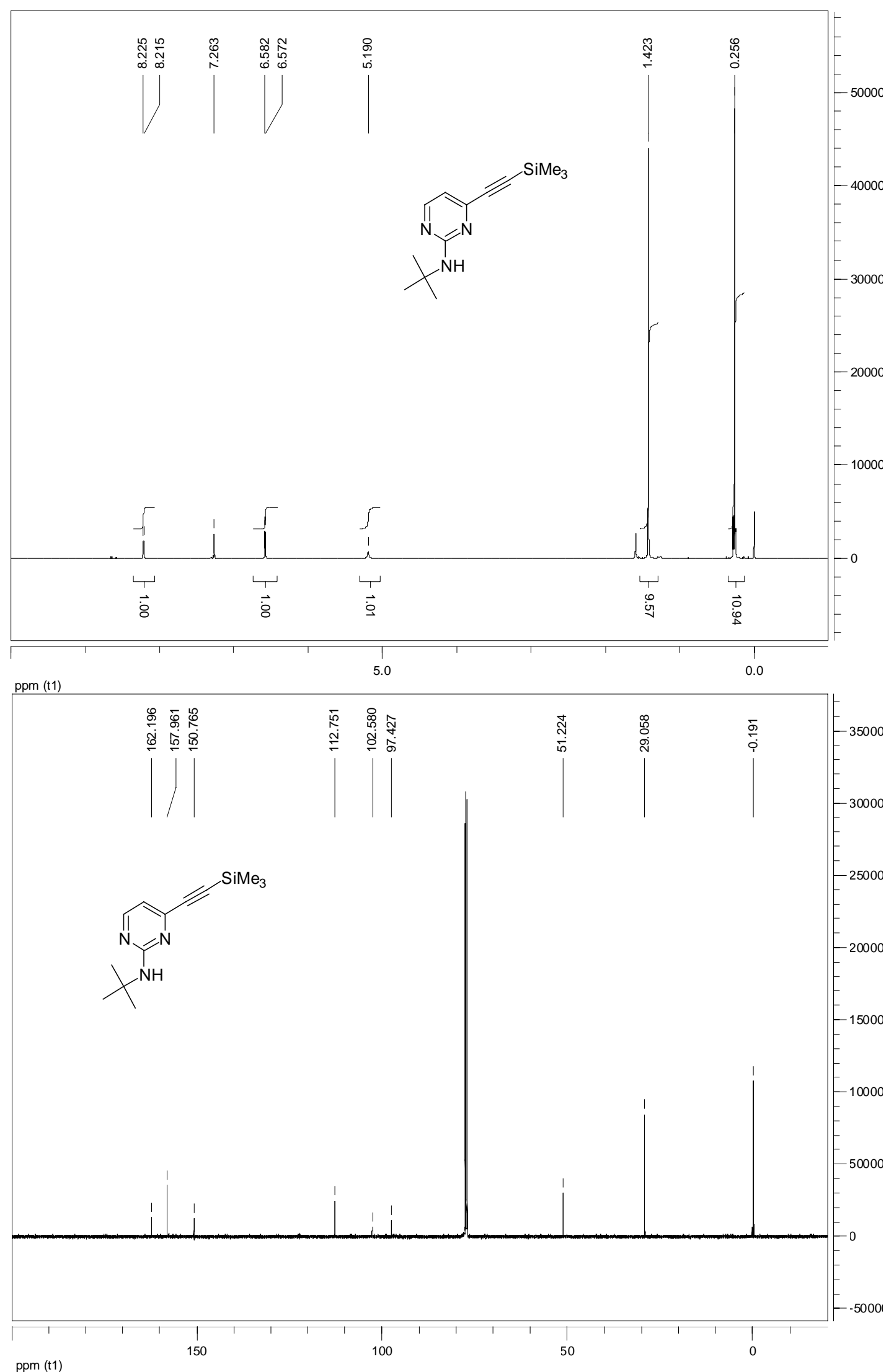



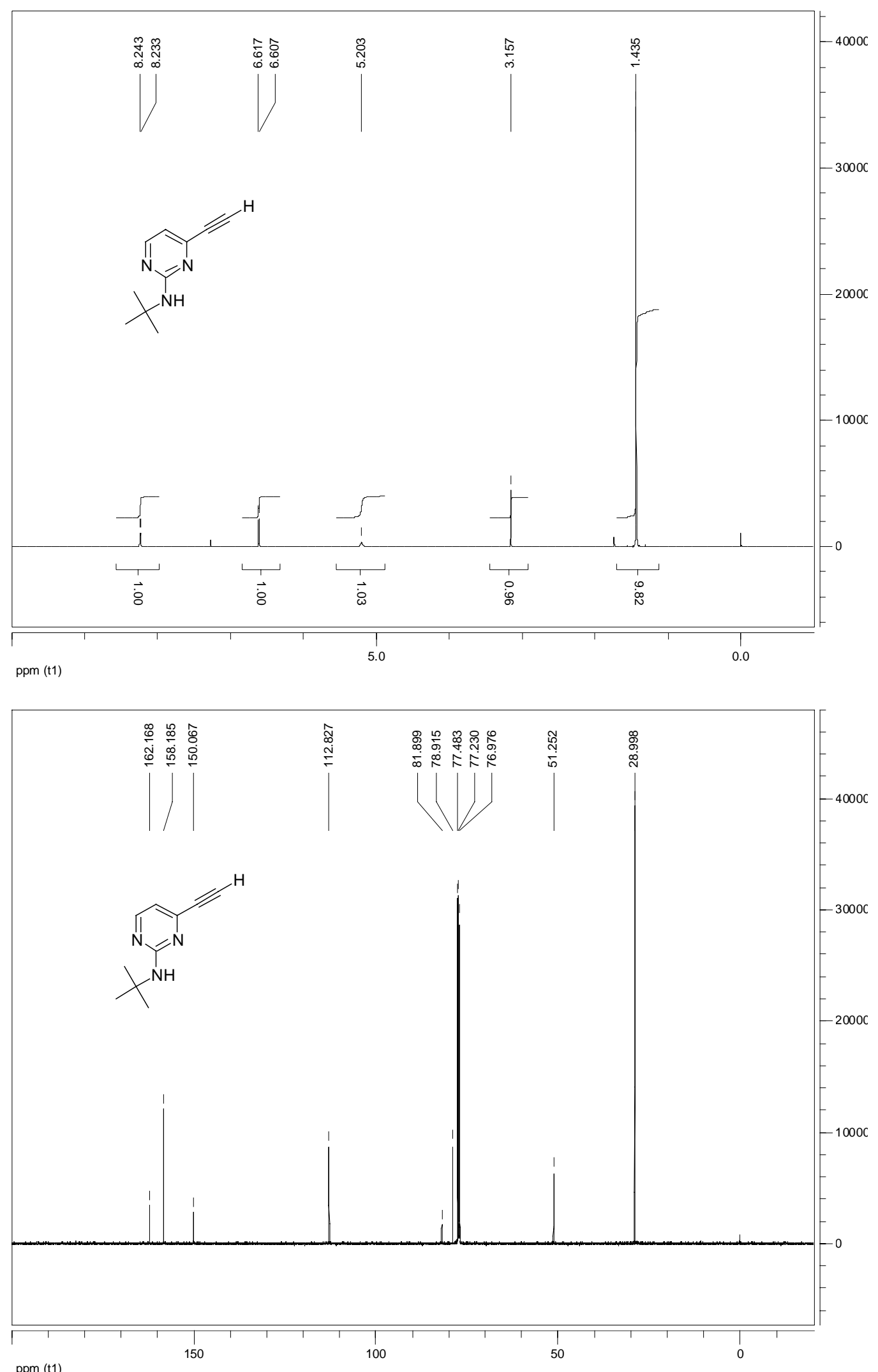

ppm (t1) 

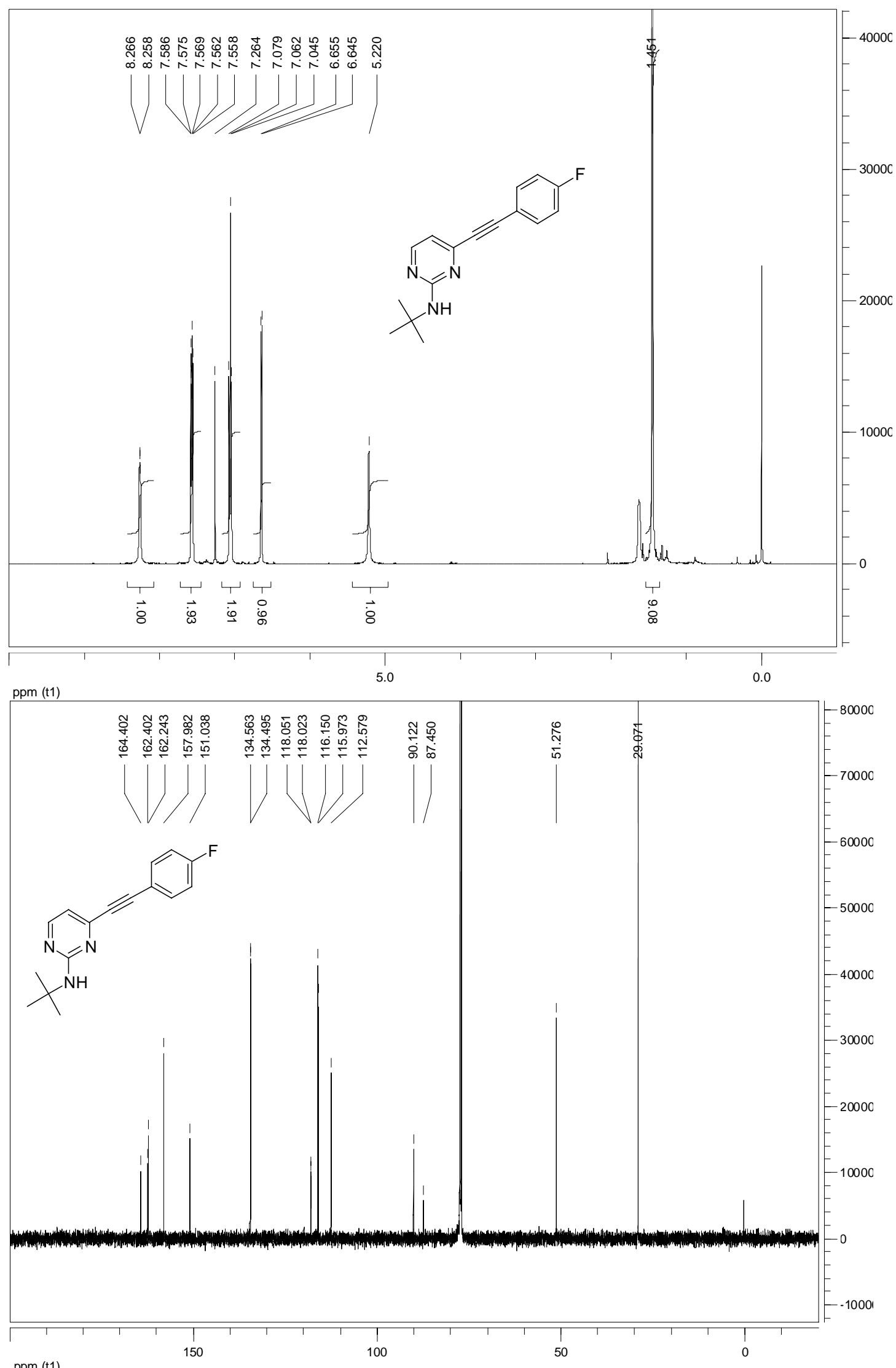

ppm (t1) 


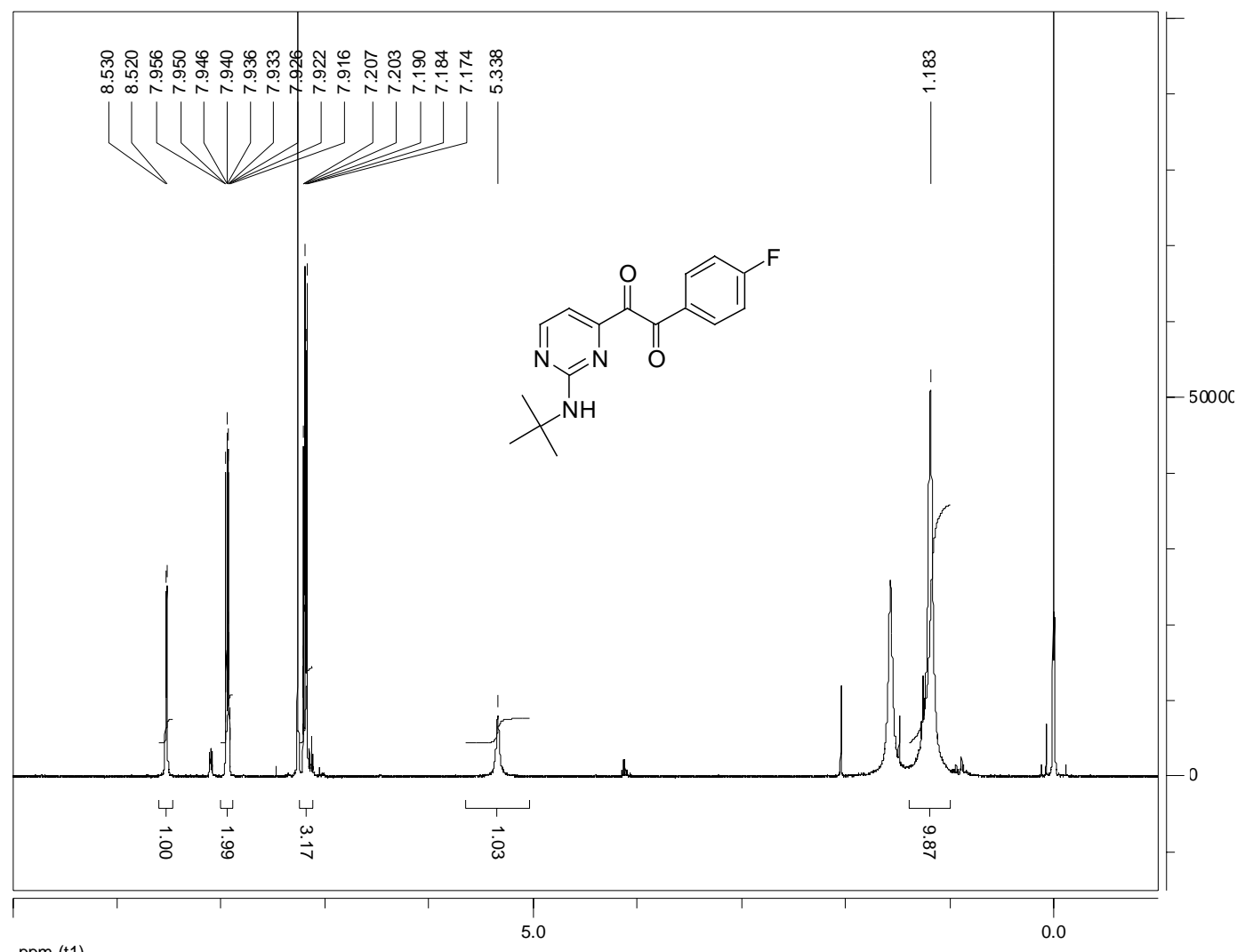

ppm (t1)

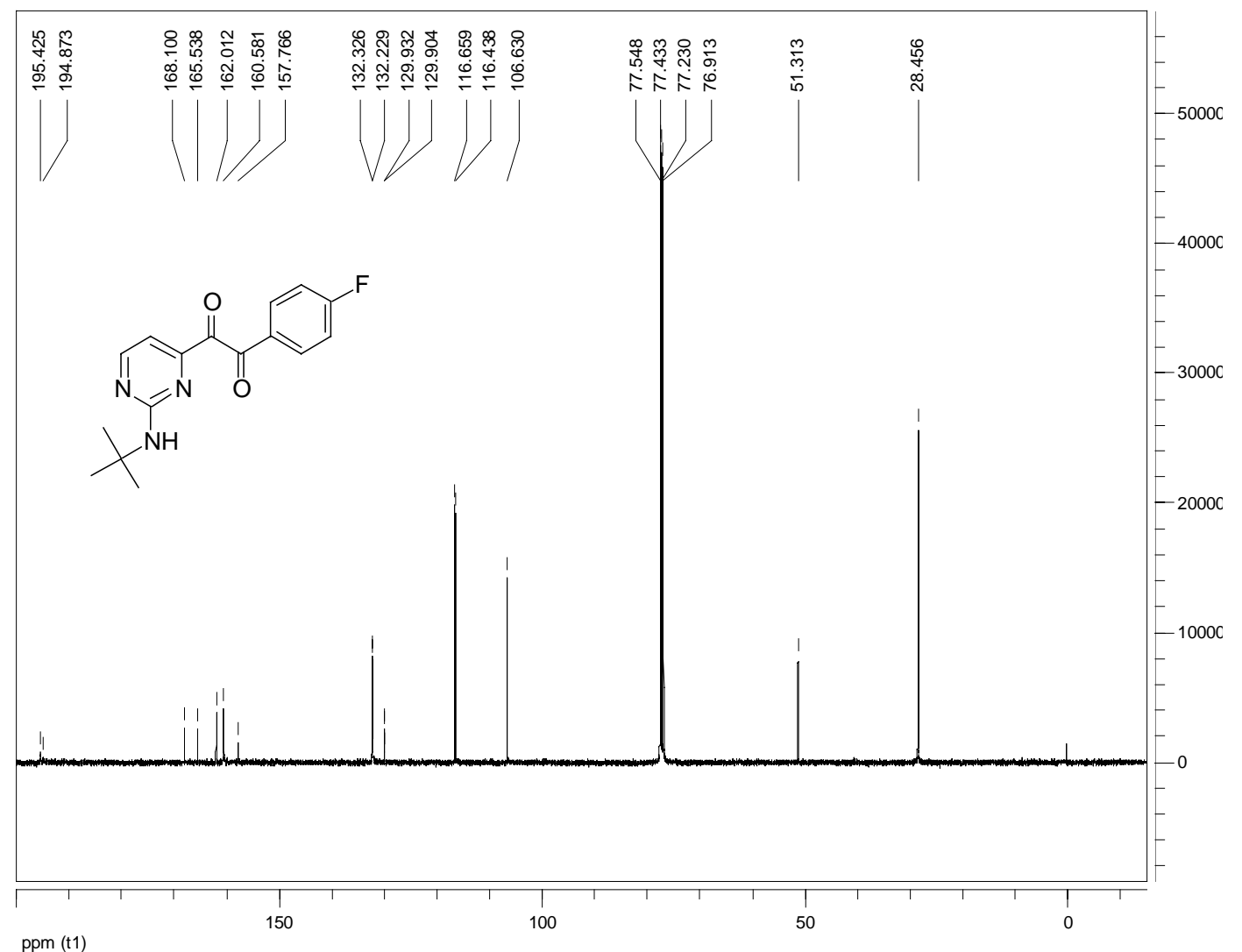



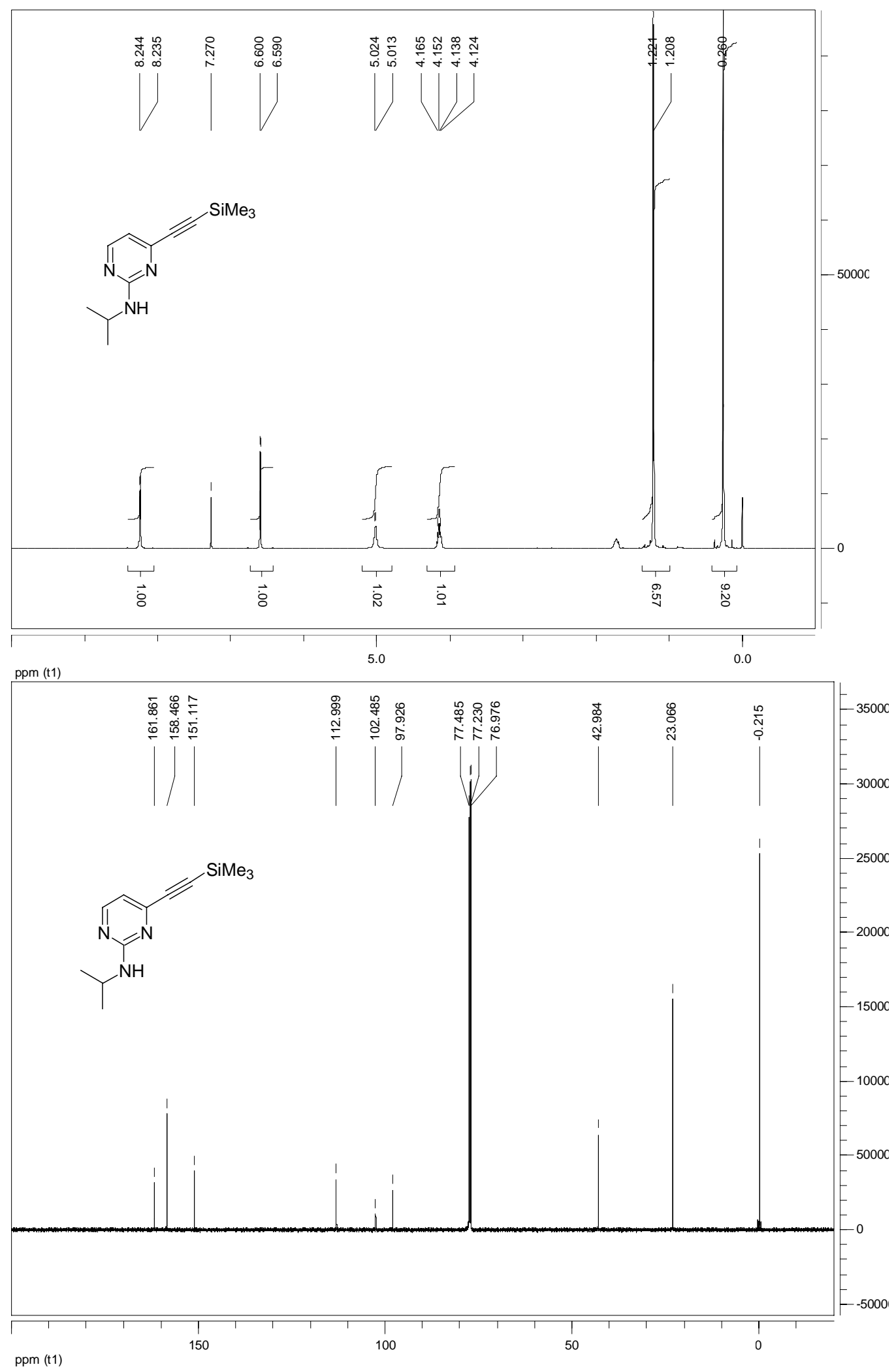


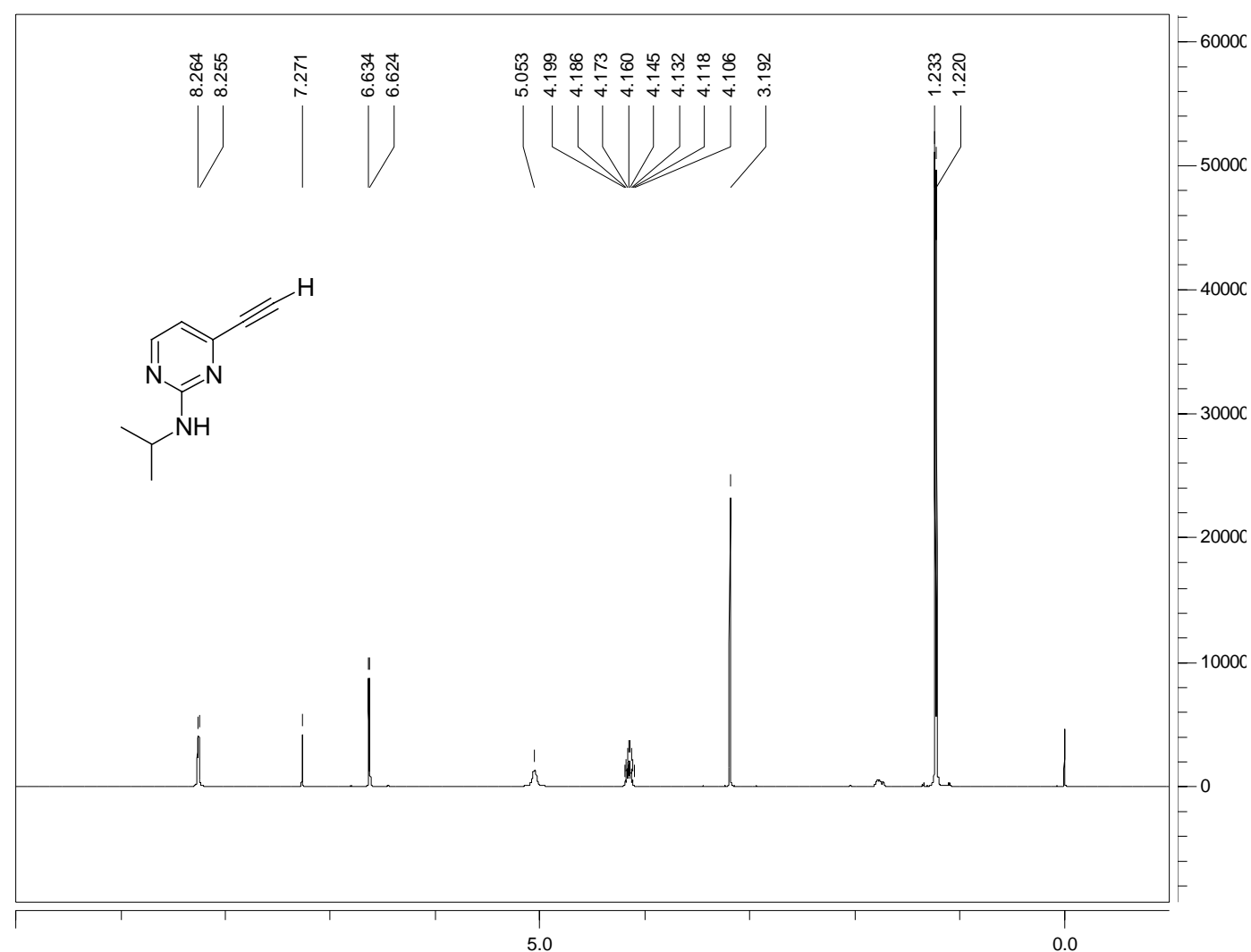

ppm (t1)

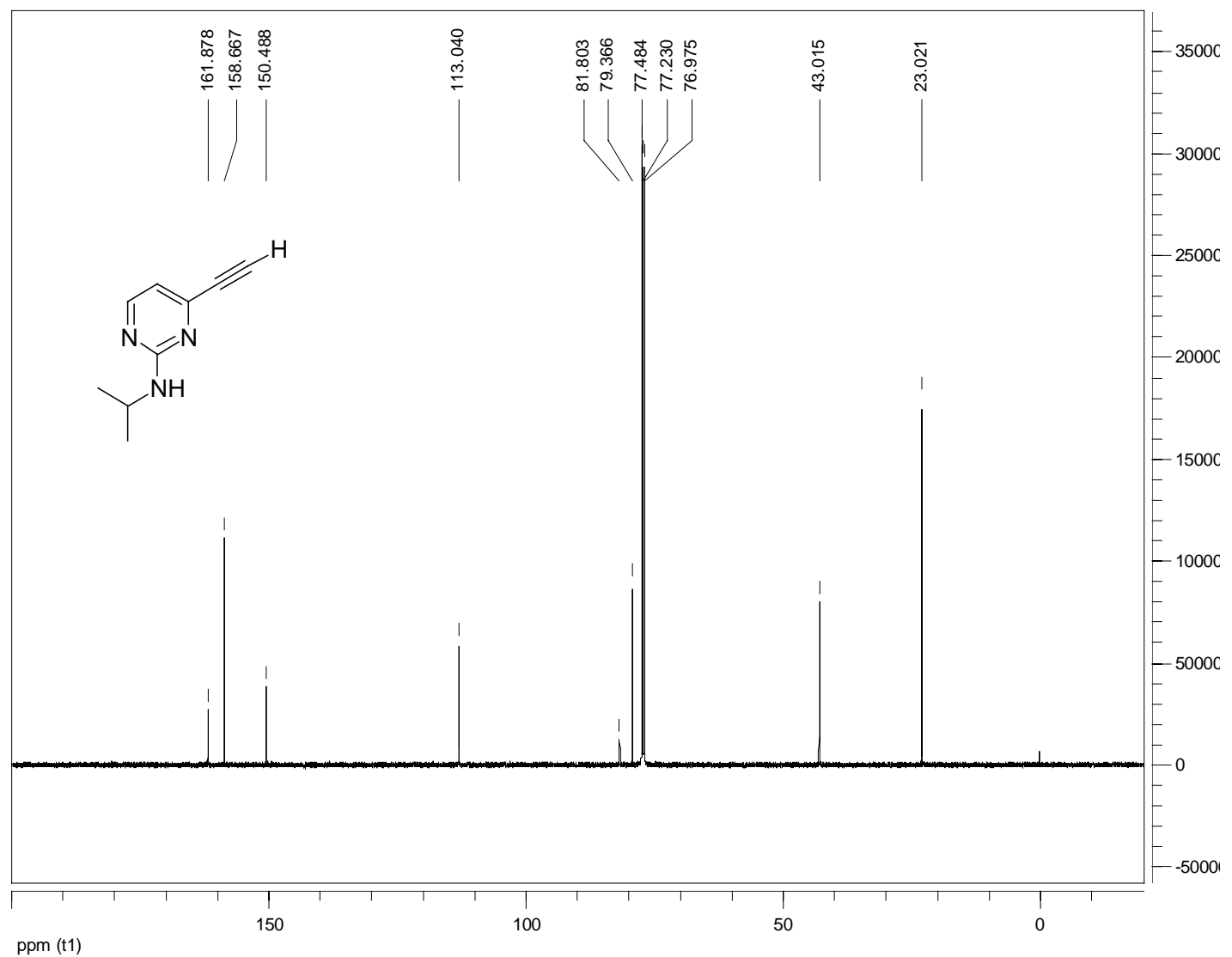

ppm (t1) 


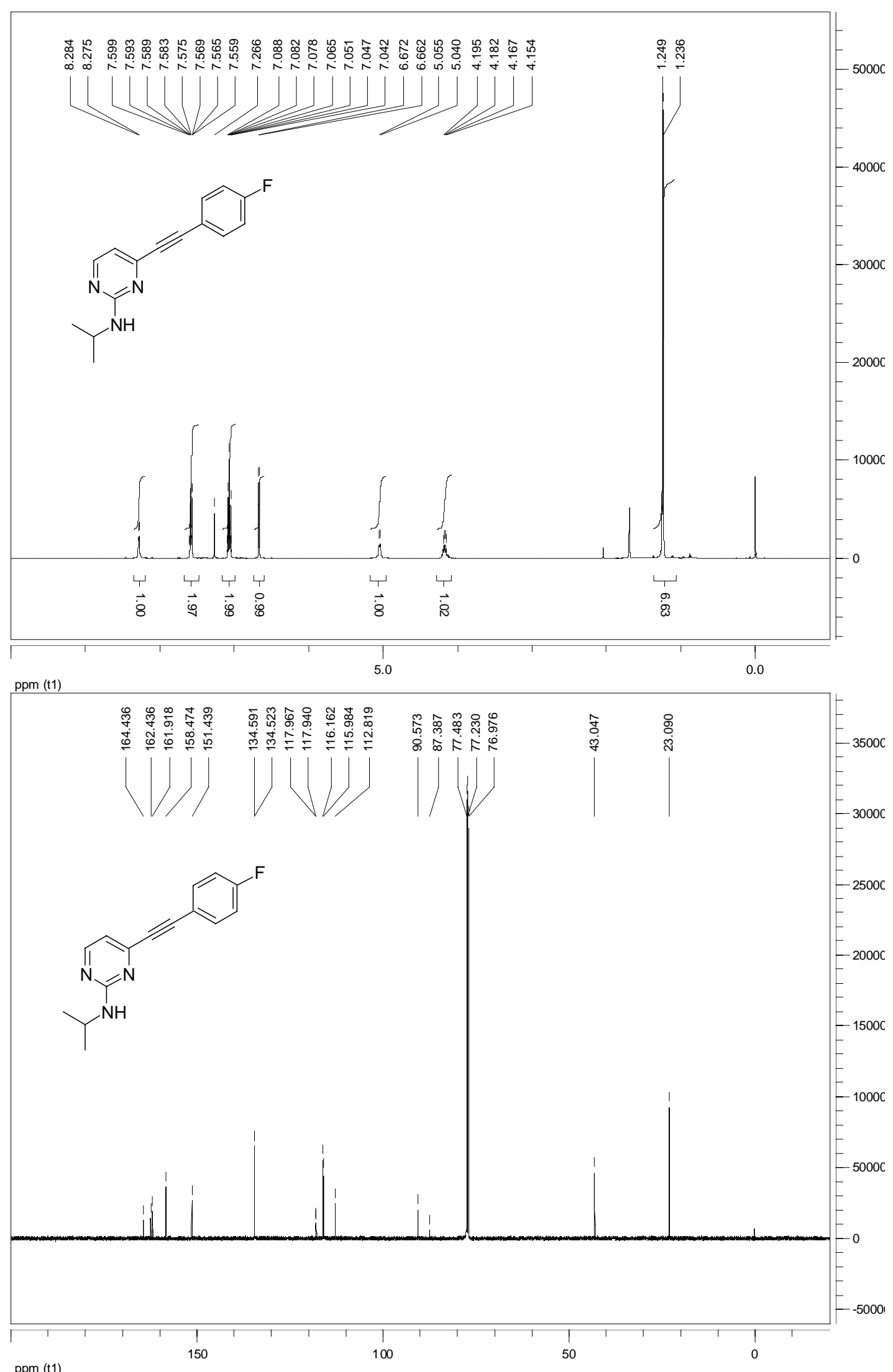




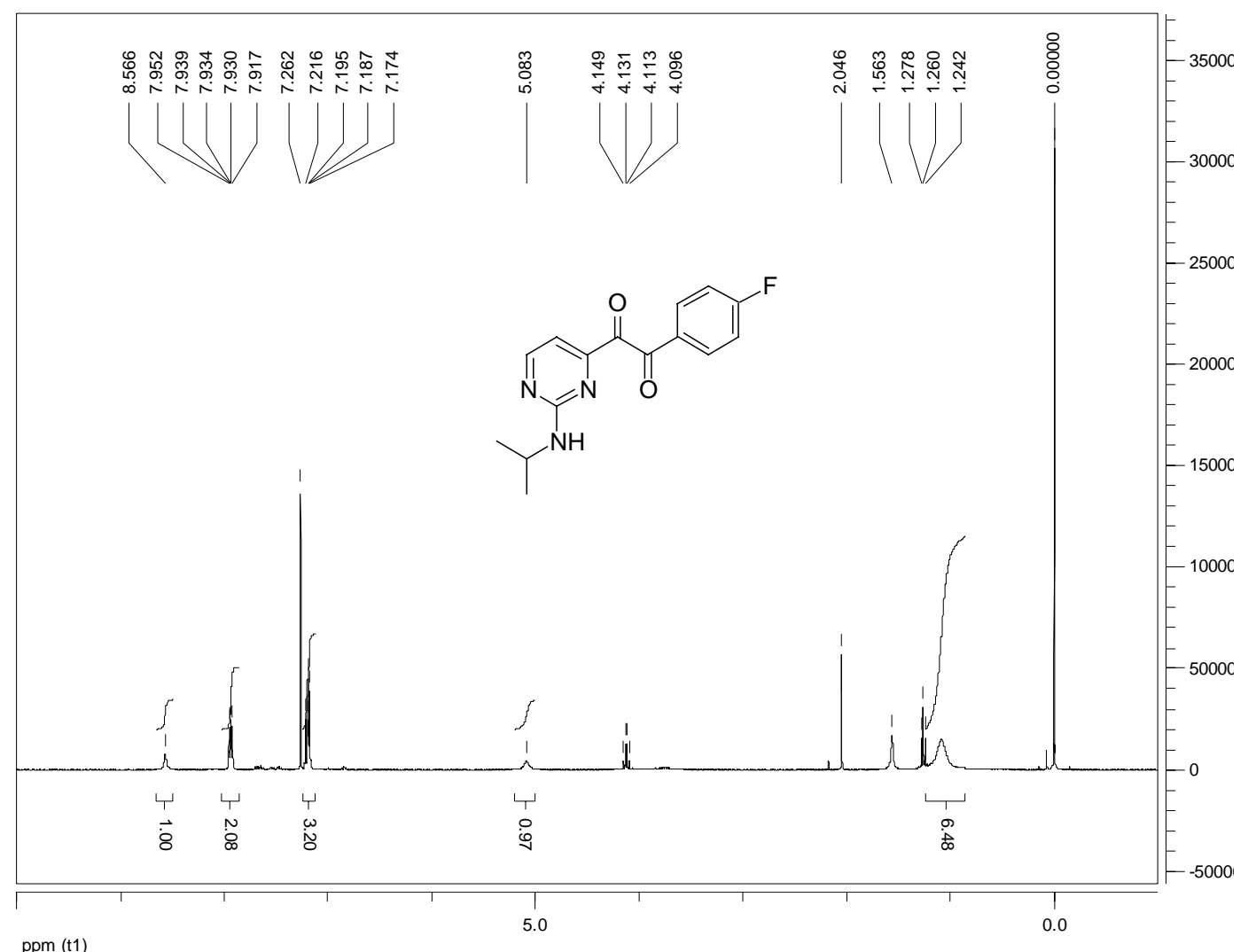

ppm (t1)

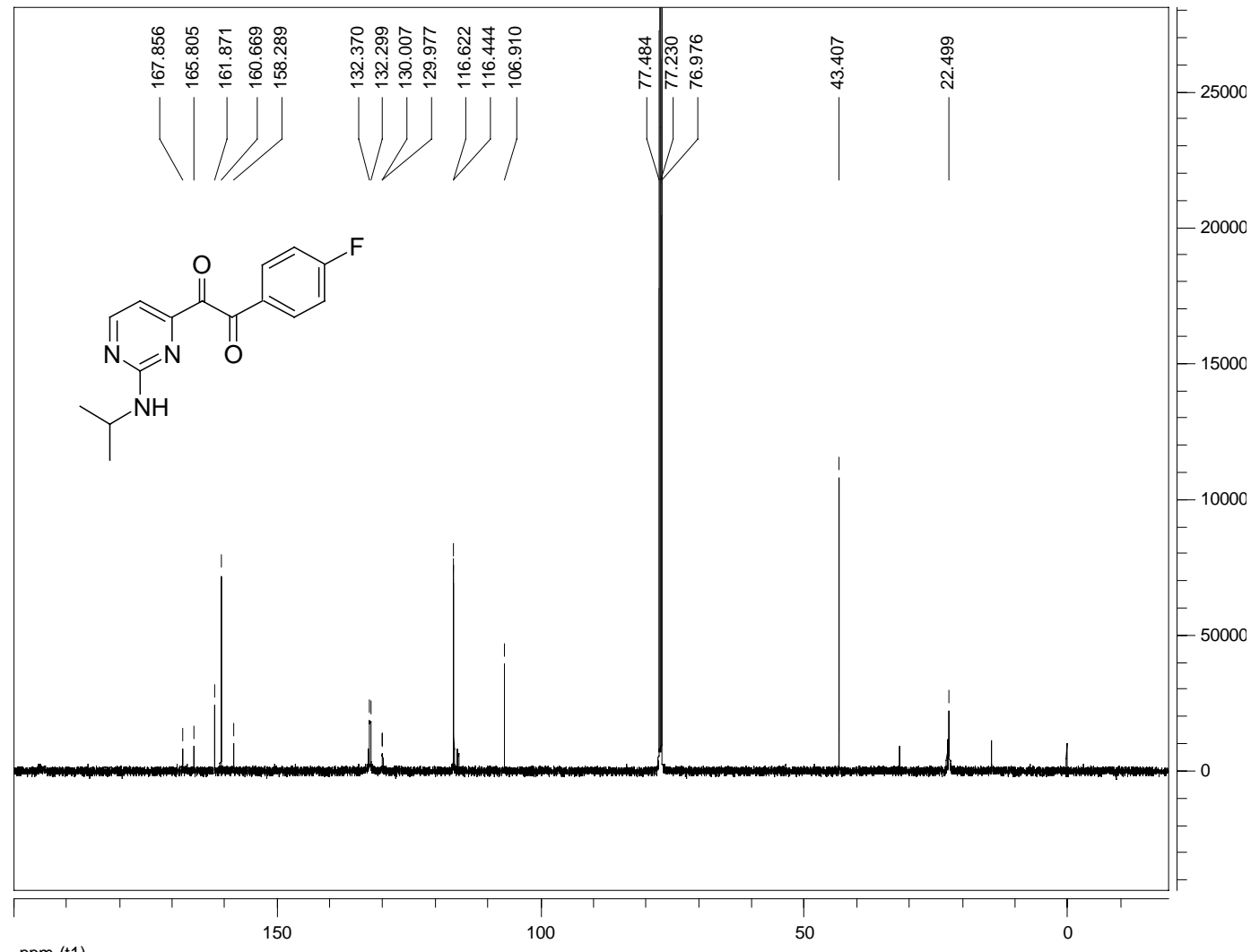

ppm (t1) 

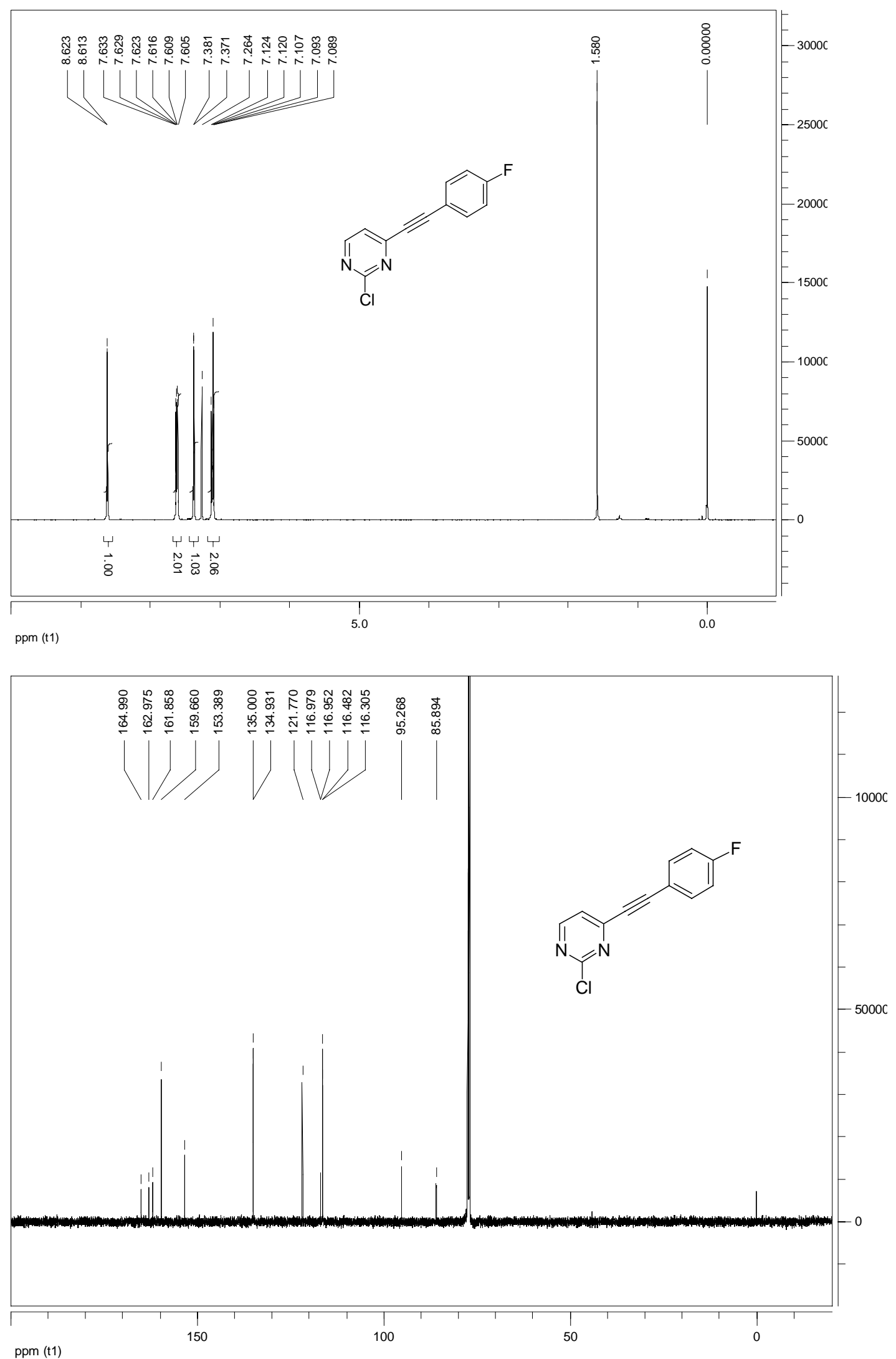


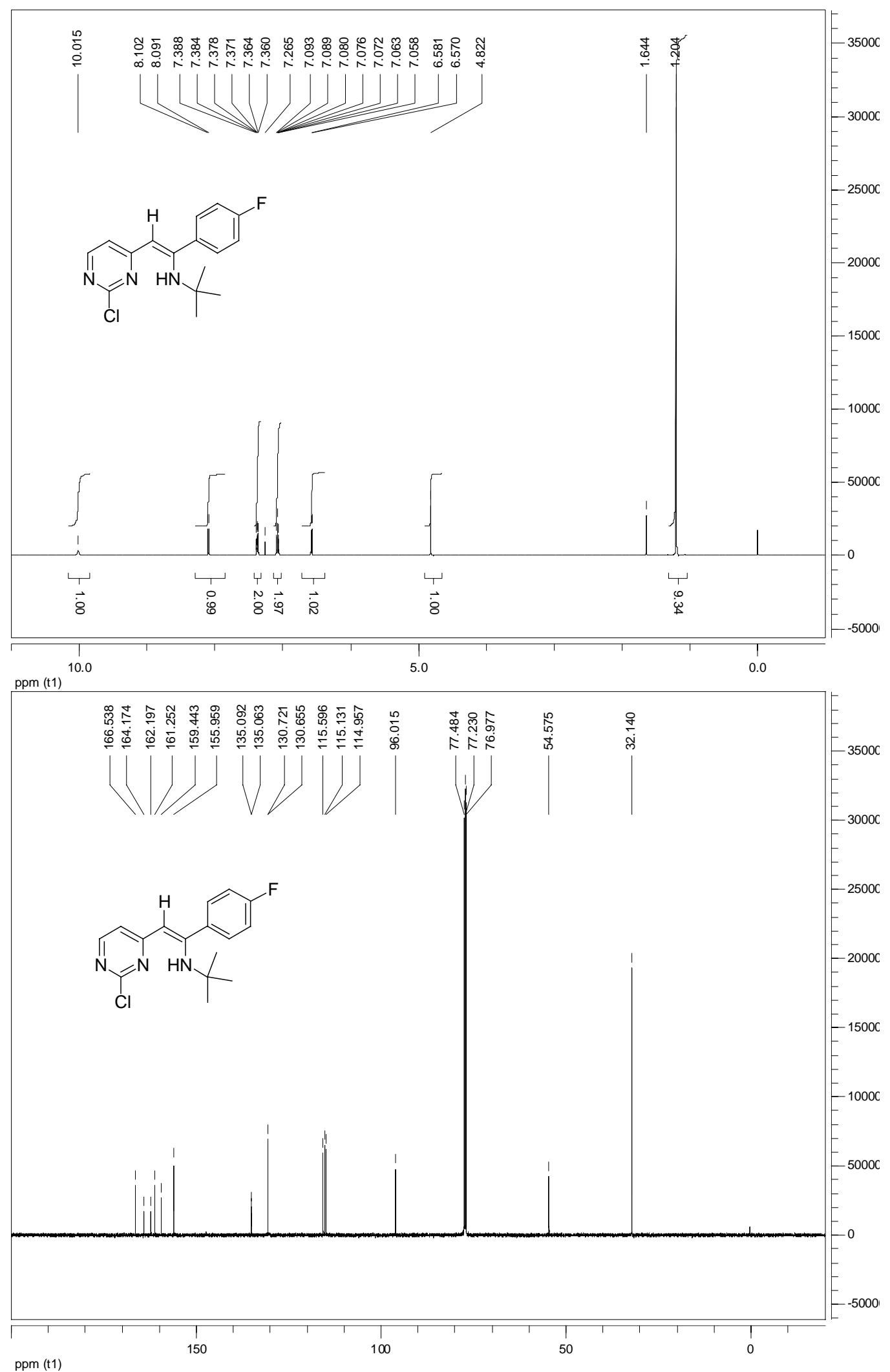




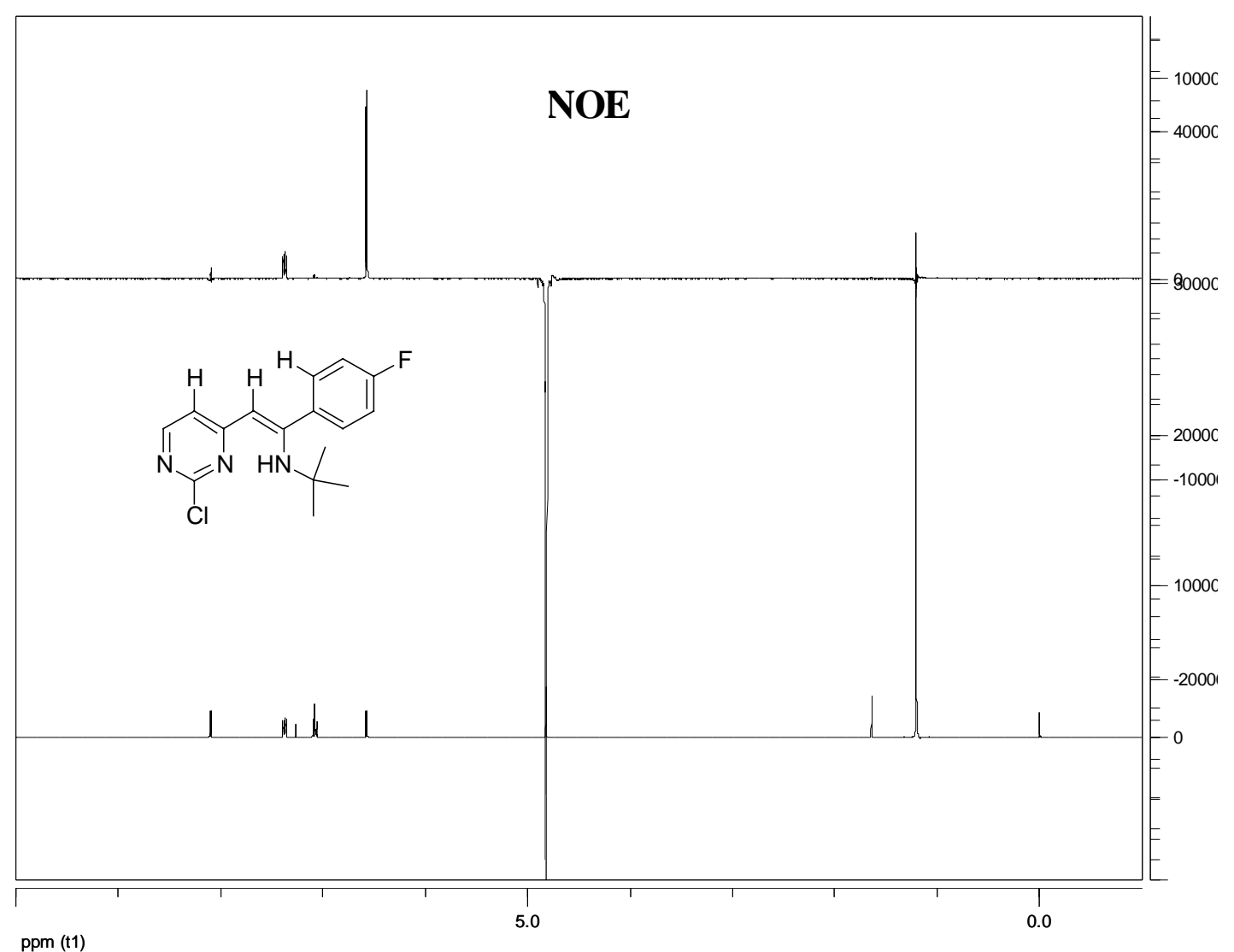

ppm (t1) 


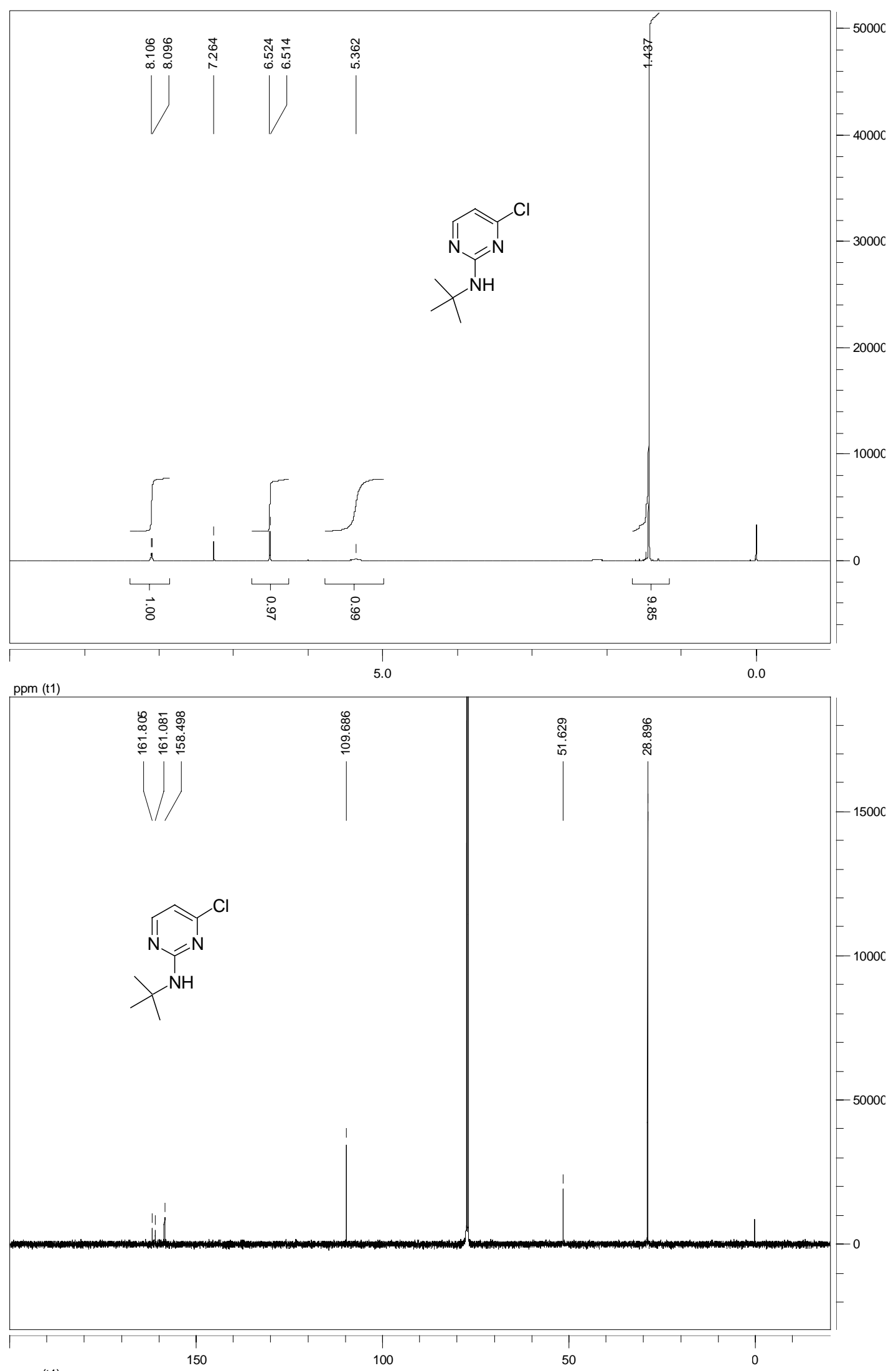

ppm (t1) 


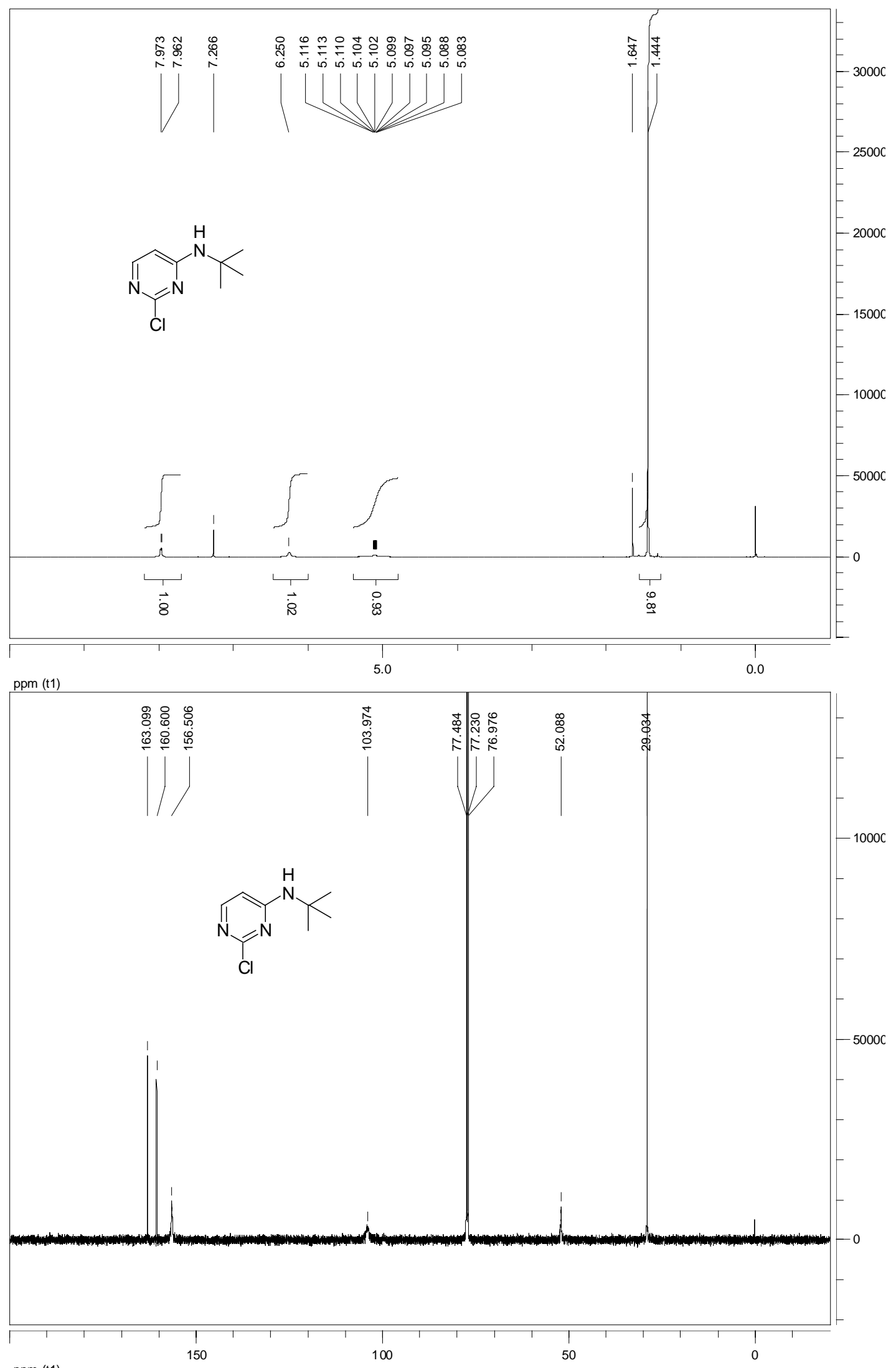

ppm (t1) 


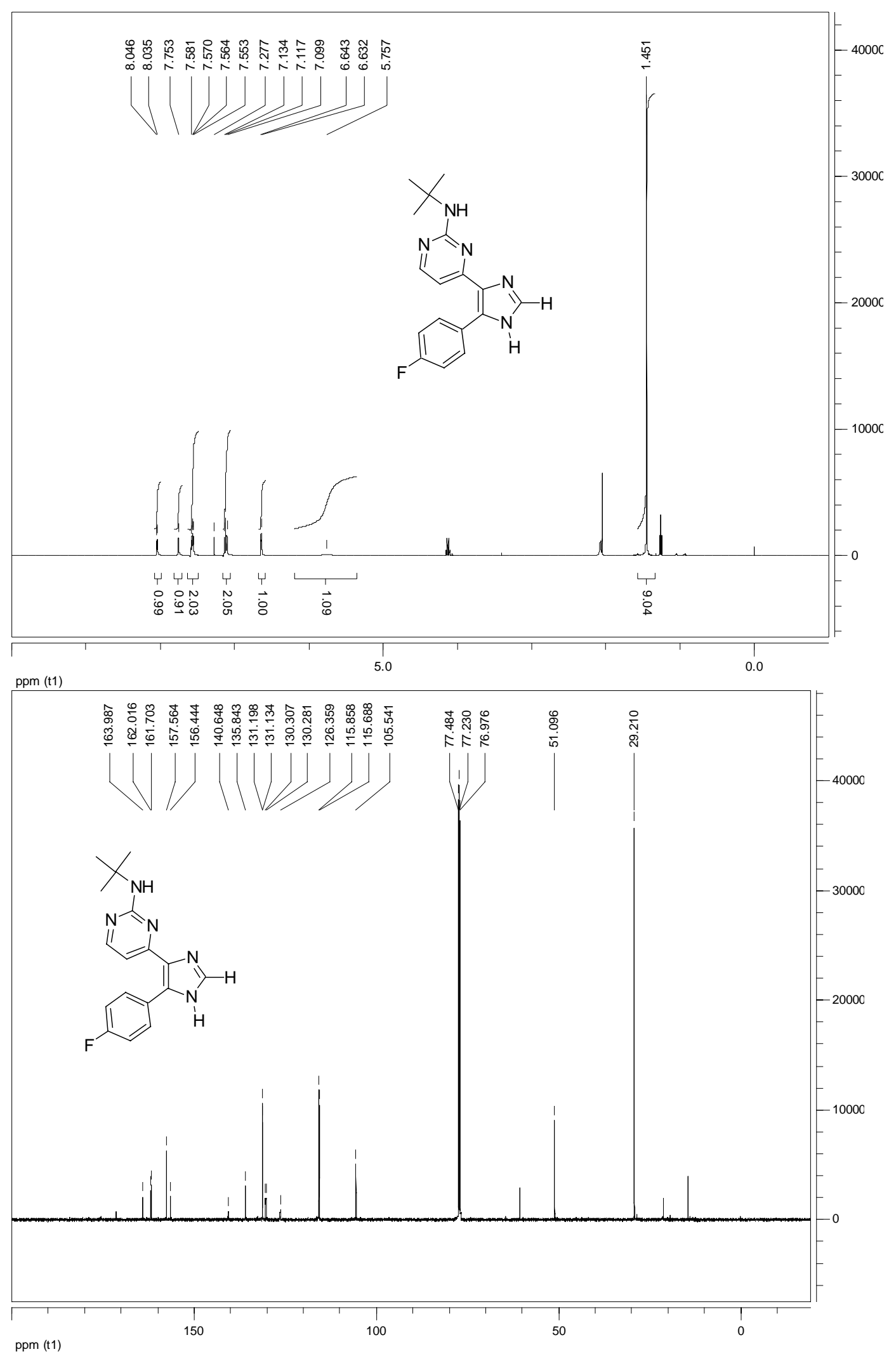



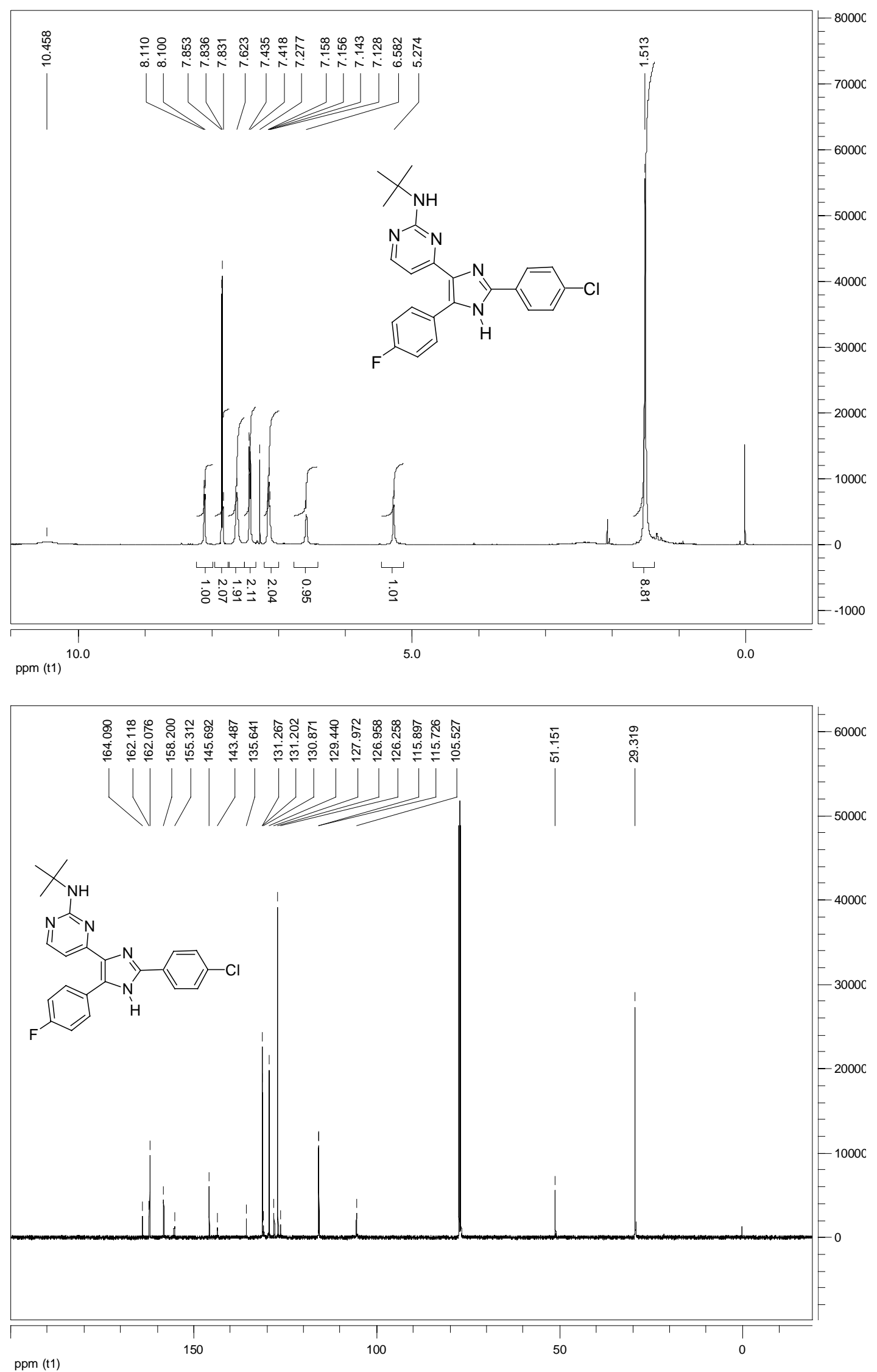

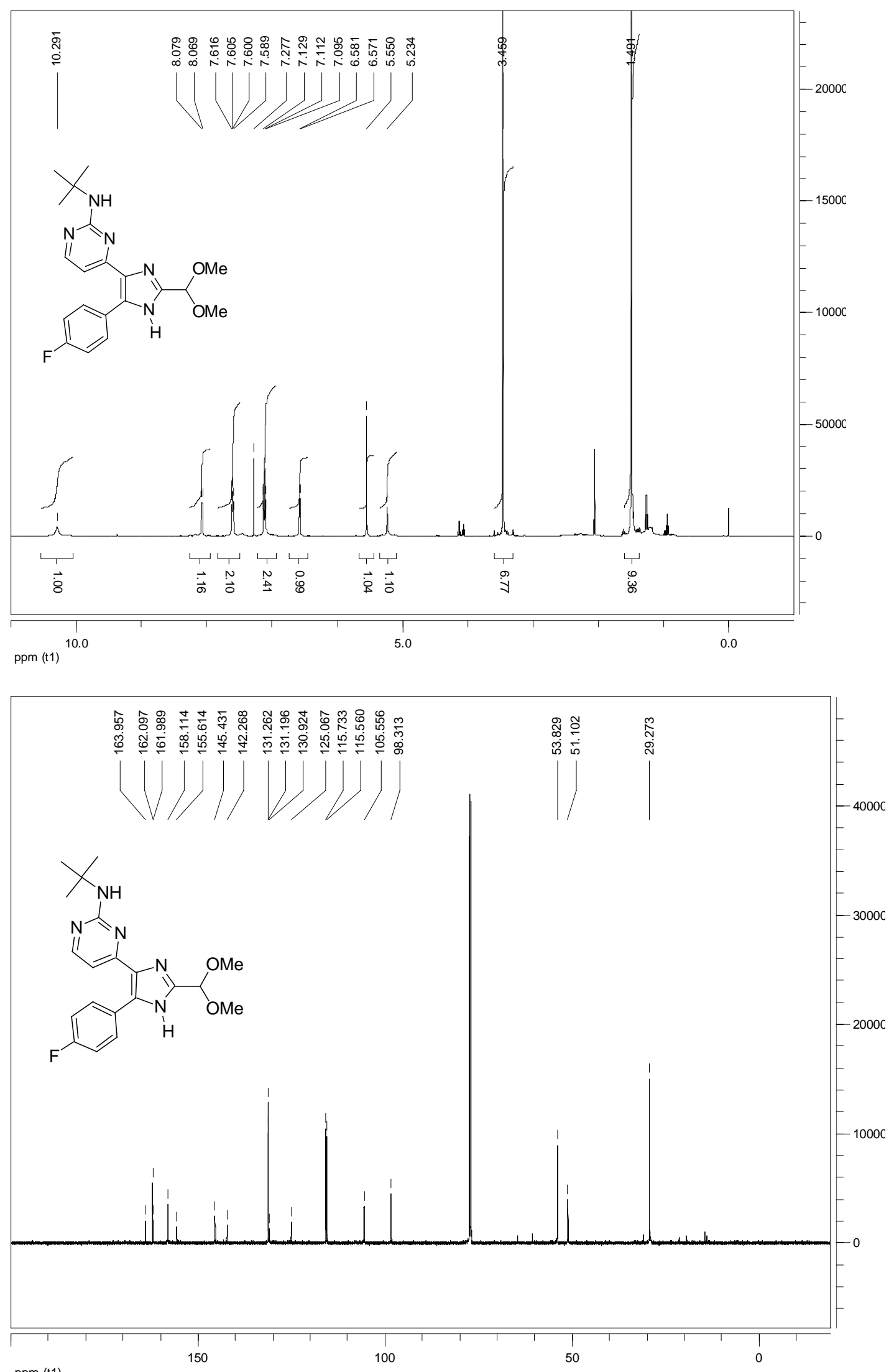

ppm (t1) 


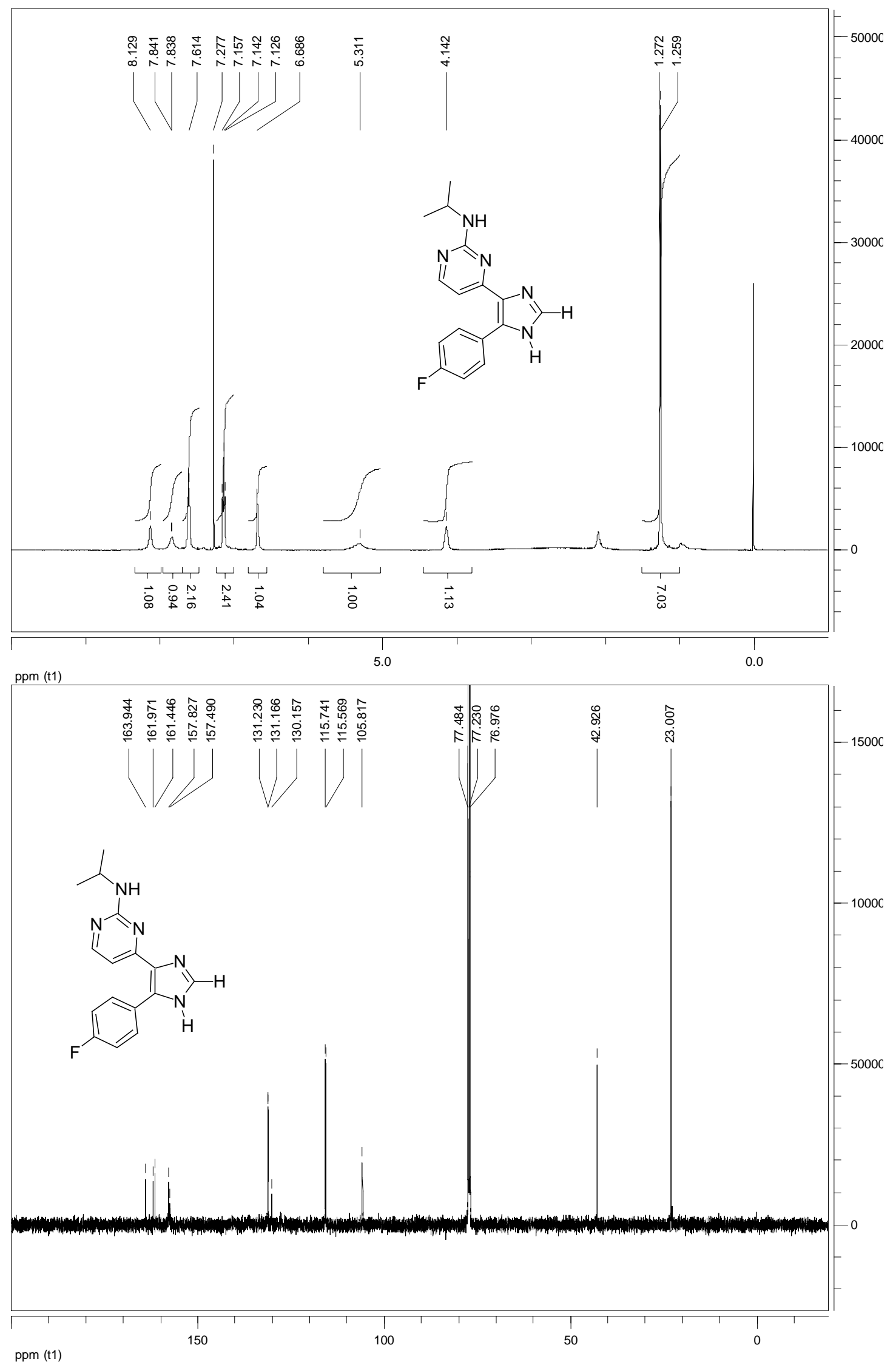




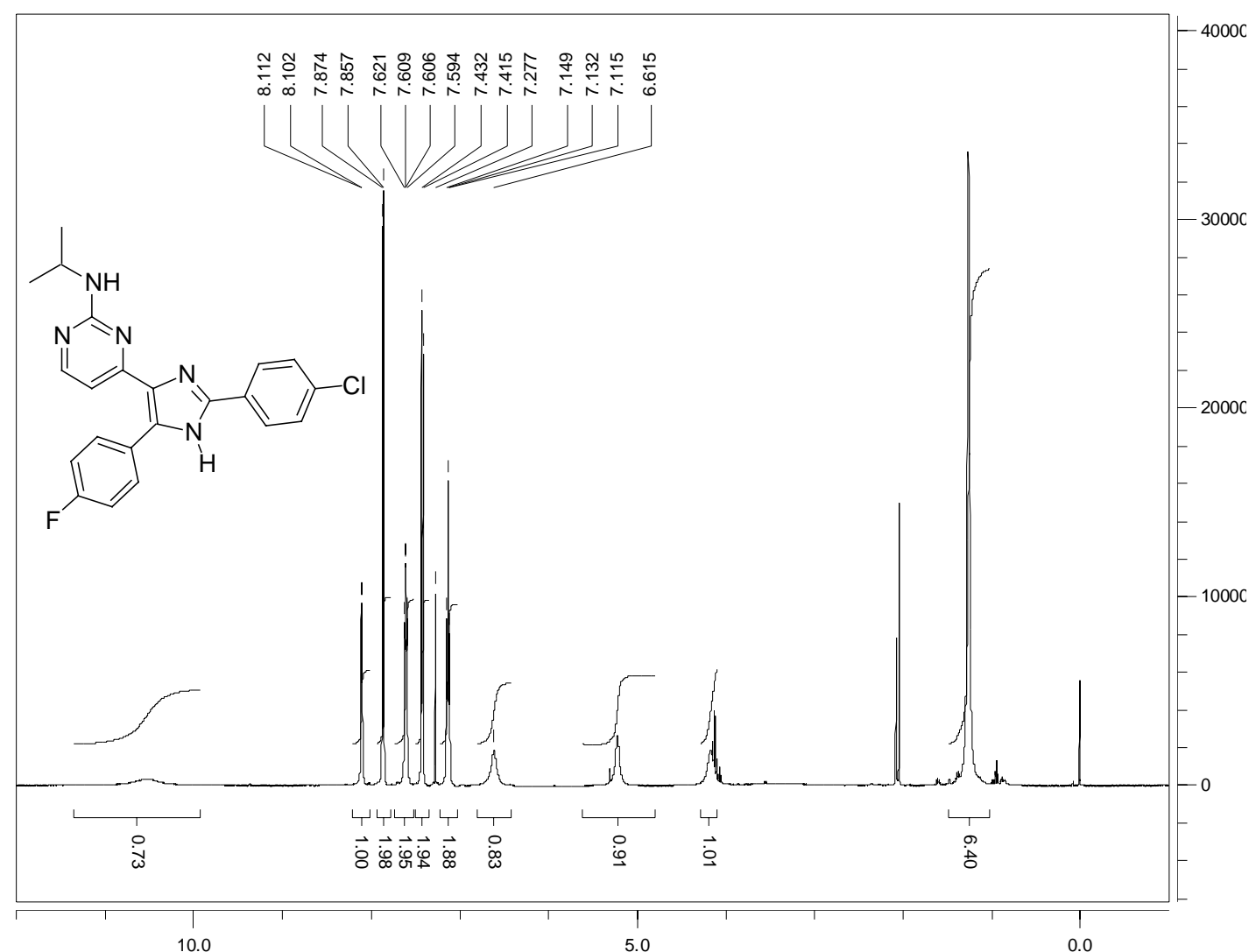

ppm (t1)

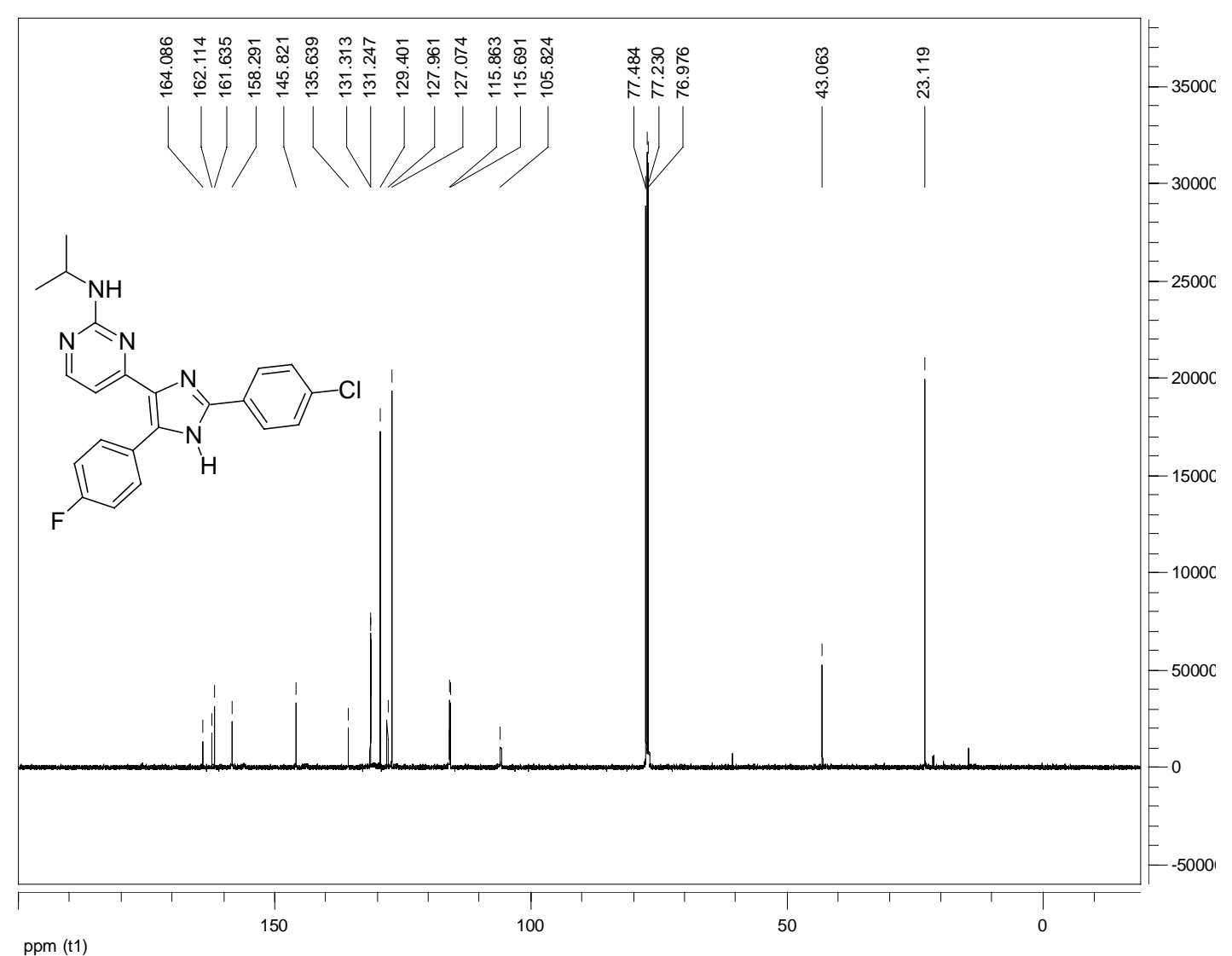

ppm (t1) 

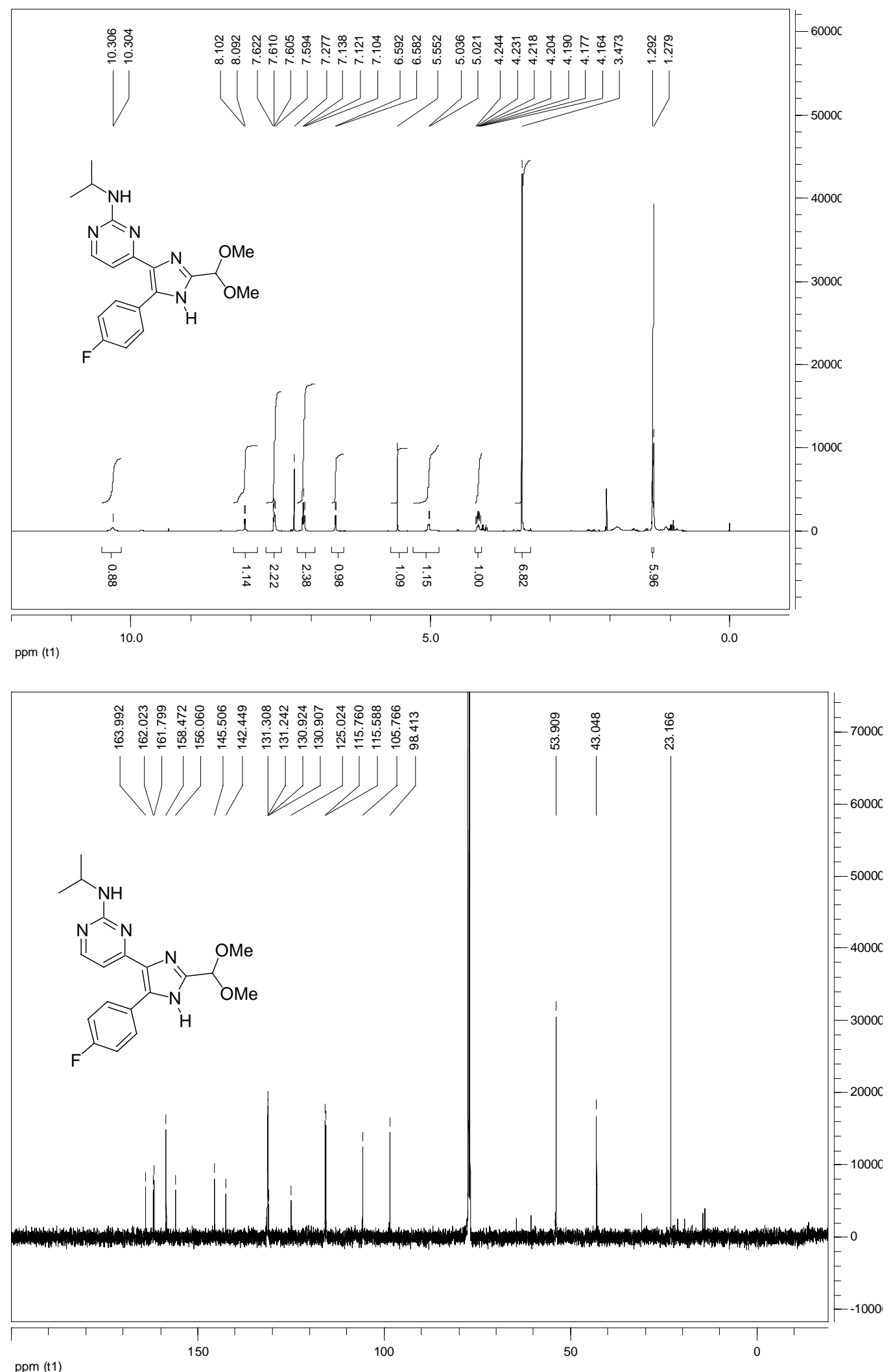